\title{
DISCRETE VECTOR POTENTIALS FOR NONSIMPLY CONNECTED THREE-DIMENSIONAL DOMAINS*
}

\author{
FRANCESCA RAPETTI ${ }^{\dagger}$, FRANÇOIS DUBOIS $^{\ddagger}$, AND ALAIN BOSSAVIT ${ }^{\S}$
}

\begin{abstract}
In this paper, we focus on the representation of a divergence-free vector field, defined, on a connected nonsimply connected domain $\Omega \subset \mathbb{R}^{3}$ with a connected boundary $\Gamma$, by its curl and its normal component on the boundary. The considered problem is discretized with $H$ (curl)- and $H$ (div)-conforming finite elements. In order to ensure the uniqueness of the vector potential, we propose a spanning tree methodology to identify the independent edges. The topological features of the domain under consideration are analyzed here by means of the homology groups of first and second order.
\end{abstract}

Key words. divergence-free vector fields, nonsimply connected domains, edge elements, discrete gauge condition, homology groups

AMS subject classification. $65 \mathrm{~N} 30$

DOI. $10.1137 /$ S0036142902412646

1. Introduction. In numerical magnetostatics, an important task is the discretization of the magnetic induction field $\mathbf{u}$, verifying the following equations:

$$
\begin{array}{cc}
\operatorname{curl} \mathbf{u}=\omega & \text { in } \Omega, \\
\operatorname{div} \mathbf{u}=0 & \text { in } \Omega, \\
\mathbf{u} \cdot \mathbf{n}_{\Gamma}=g & \text { on } \Gamma,
\end{array}
$$

where $\Omega$ is an open subset of $\mathbb{R}^{3}, \Gamma$ is its boundary, $\mathbf{n}_{\Gamma}$ is the outward going normal to $\Gamma, \omega$ is a given current density, and $g$ is a scalar function defined on $\Gamma$. A conforming or nonconforming discretization that respects (2) is difficult to obtain with the finite element method [19]. On the other hand, a way to exactly satisfy (2) is to represent $\mathbf{u}$ in terms of a vector potential, i.e., a field $\mathbf{p}$ such that

$$
\mathbf{u}=\operatorname{curl} \mathbf{p} .
$$

The vector $\mathbf{p}$ is not unique but defined up to the gradient of a scalar function. A classical way to ensure the uniqueness of $\mathbf{p}$ is to prescribe a gauge condition such as the Coulomb gauge

$$
\operatorname{div} \mathbf{p}=0
$$

and suitable boundary conditions. Moreover, different choices of boundary conditions for the vector field $\mathbf{p}$ are possible, and we refer to $[3,4]$ for existence and uniqueness results. The vector potential is just a tool for representing the field $\mathbf{u}$ and must be

\footnotetext{
* Received by the editors August 3, 2002; accepted for publication (in revised form) March 13, 2003; published electronically September 17, 2003.

http://www.siam.org/journals/sinum/41-4/41264.html

†Laboratoire de Mathématiques, UMR 6621 CNRS, Université de Nice et Sophia-Antipolis, Parc Valrose, 06108 Nice cedex 02, France (frapetti@math.unice.fr).

${ }^{\ddagger}$ Laboratoire des Applications Scientifiques du Calcul Intensif, UPR 9029 CNRS, Université Paris Sud, Bâtiment 506, 91403 Orsay, France (dubois@asci.fr).

$\S$ Division Recherche et Développement, Electricité de France, 1 Avenue du Général de Gaulle, 92141 Clamart cedex, France (Alain.Bossavit@der.edfgdf.fr).
} 
easily computable under some constraints on $\mathbf{u}$. In this paper, we choose to fix the current density $\omega$ and the magnetic induction flux $g$ across the entire boundary. We remark that, if (2) and (3) are satisfied, then the mean value of the function $g$ across the boundary of $\Omega$ is necessarily equal to zero; i.e., $\int_{\Gamma} g d \Gamma=0$. The chosen problem can be also read in the framework of fluid dynamics: for a given vorticity $\omega$ in $\Omega$ and mass inflow $g$ across the boundary $\Gamma$ of $\Omega$, we look for a velocity field $\mathbf{u}$ that satisfies (1) and (3) in the incompressible case, i.e., under the constraint (2).

We restrict ourselves to the previous problem in a nonsimply connected threedimensional domain with a connected boundary. For the analysis of the mixed formulation of a similar problem in simply connected domains with a nonconnected boundary, see [10].

Concerning the outline of the paper, in section 2, after recalling classical results on vector fields, we split the linear problem in $\Omega$ into a homogeneous problem in $\Omega$ (i.e., $g=0$ ) and a problem on the boundary of $\Omega$ (i.e., $g \neq 0$ ). Then a concrete construction method of a vector potential $\mathbf{p}$ from only the data $\omega$ and $g$ is presented. In the discretized problem, the compatibility between these two subproblems requires that the discrete field $\mathbf{p}_{m}$ associated with a mesh $m$ in $\Omega$ has a tangential component on each point of the boundary. The major difficulty is the definition of a "good" discrete space which guarantees the existence of the discrete potential. We start with a short introduction on the homology groups in sections 3 and 4 . Then in sections 5 and 6 , we adapt the discrete gauge initially proposed by a team of the École Polytechnique $[10,28]$ to the case of simply connected domains $\Omega \subset \mathbb{R}^{3}$. Developing the problem presented in [11] in more detail, we generalize in section 7 to the case of proposed nonsimply connected domains. We finally end in section 8 by a short overview on the adopted algorithms and their application in the case where $\Omega$ is a torus.

Let us introduce some notation. We consider $\Omega$ as a connected bounded domain of $\mathbb{R}^{3}$, with a connected regular boundary $\Gamma$. The scalar product between two vectors $\mathbf{a}, \mathbf{b}$ defined in $\Omega$ is denoted by $\mathbf{a} \cdot \mathbf{b}$, whereas their vector product is denoted by $\mathbf{a} \times \mathbf{b}$. The tangential component of a vector $\mathbf{v}$ on $\Gamma$ is $\pi \mathbf{u}=\left(\mathbf{n}_{\Gamma} \times \mathbf{u}\right) \times \mathbf{n}_{\Gamma}$, and we have a Green formula for regular vector fields $\mathbf{u}$ and $\mathbf{v}$ that reads

$$
\int_{\Omega} \mathbf{v} \cdot \operatorname{curl} \mathbf{u}=\int_{\Omega} \operatorname{curl} \mathbf{v} \cdot \mathbf{u}+\int_{\Gamma}\left(\mathbf{n}_{\Gamma} \times \mathbf{v}\right) \cdot \mathbf{u} .
$$

The following operators are defined on $\Gamma$ as in [9] as follows:

- the surface gradient, $\operatorname{grad}_{\Gamma} u$, and surface $\operatorname{curl}, \operatorname{curl}_{\Gamma} u$, of a scalar function $u$ defined on $\Gamma: \operatorname{curl}_{\Gamma} u=\operatorname{grad}_{\Gamma} u \times \mathbf{n}_{\Gamma}$;

- the surface curl, $\operatorname{curl}_{\Gamma} \mathbf{v}$, and surface divergence, $\operatorname{div}_{\Gamma} \mathbf{v}$, of a tangential vector function $\mathbf{v}$ defined on $\Gamma: \operatorname{div}_{\Gamma} \mathbf{v}=\operatorname{curl}_{\Gamma}\left(\mathbf{n}_{\Gamma} \times \mathbf{v}\right)$.

By duality, these operators are also defined for a scalar $t$ or vector $\mathbf{w}$ distributions on $\Gamma$ as follows:

$$
\begin{aligned}
& \left\langle\operatorname{grad}_{\Gamma} t, \mathbf{v}\right\rangle_{\Gamma}=-\left\langle t, \operatorname{div}_{\Gamma} \mathbf{v}\right\rangle_{\Gamma} \quad \forall \mathbf{v}, \\
& \left\langle\operatorname{curl}_{\Gamma} t, \mathbf{v}\right\rangle_{\Gamma}=\left\langle t, \operatorname{curl}_{\Gamma} \mathbf{v}\right\rangle_{\Gamma} \quad \forall \mathbf{v}, \\
& \left\langle\operatorname{curl}_{\Gamma} \mathbf{w}, u\right\rangle_{\Gamma}=\left\langle\mathbf{w}, \operatorname{curl}_{\Gamma} u\right\rangle_{\Gamma} \quad \forall u, \\
& \left\langle\operatorname{div}_{\Gamma} \mathbf{w}, u\right\rangle_{\Gamma}=\left\langle\mathbf{w}, \operatorname{grad}_{\Gamma} u\right\rangle_{\Gamma} \quad \forall u .
\end{aligned}
$$

Here, the duality product $\langle\cdot, \cdot\rangle_{\Gamma}$ of two vectors is the scalar product on $\Gamma[9]$ :

$$
\langle\mathbf{w}, \mathbf{v}\rangle_{\Gamma}=\int_{\Gamma} \mathbf{w} \cdot \mathbf{v}
$$


The Sobolev spaces $L^{2}(\Omega), H^{1}(\Omega)$ are Hilbert spaces with their natural norms $\|.\|_{0, \Omega}$ and $\|.\|_{1, \Omega}$, respectively [1]. Following [15], we define

$$
\begin{aligned}
& H(\operatorname{div}, \Omega)=\left\{\mathbf{u} \in L^{2}(\Omega)^{3} \mid \operatorname{div} \mathbf{u} \in L^{2}(\Omega)\right\}, \\
& H(\operatorname{curl}, \Omega)=\left\{\mathbf{u} \in L^{2}(\Omega)^{3} \mid \operatorname{curl} \mathbf{u} \in L^{2}(\Omega)^{3}\right\},
\end{aligned}
$$

and associated norms $\|.\|_{\operatorname{div}, \Omega}$ and $\|.\|_{\operatorname{curl}, \Omega}$. We also need to introduce

$$
\begin{aligned}
& H\left(\operatorname{div}_{0}, \Omega\right)=\{\mathbf{u} \in H(\operatorname{div}, \Omega) \mid \operatorname{div} \mathbf{u}=0\} \\
& H_{0}(\operatorname{div}, \Omega)=\left\{\mathbf{u} \in H(\operatorname{div}, \Omega) \mid \mathbf{u} \cdot \mathbf{n}_{\Gamma}=0\right\} \\
& H_{0}(\operatorname{curl}, \Omega)=\left\{\mathbf{u} \in H(\operatorname{curl}, \Omega) \mid \mathbf{u} \times \mathbf{n}_{\Gamma}=\mathbf{0}\right\} \\
& L_{0}^{2}(\Omega)=\left\{u \in L^{2}(\Omega) \mid \int_{\Omega} u=0\right\} \\
& \mathcal{C}^{1,1}(\bar{\Omega})=\left\{u \in \mathcal{C}^{1}(\bar{\Omega}) \mid \operatorname{grad} u \text { is a vector of Lipschitz functions }\right\} .
\end{aligned}
$$

In a few words, a domain $\Omega$ is of class $\mathcal{C}^{1,1}$ if it admits a representation through a $\mathcal{C}^{1,1}(\bar{\Omega})$ map $[15]$. Note that the boundary of such a domain has a normal vector almost everywhere. In the following, given a space $S$, the notation $\operatorname{dim}[S]$ denotes the dimension of $S$. If $S$ is a set, its cardinality, i.e., the number of its elements, is denoted by $\# S$.

2. The continuous problem. We are interested in the following problem: given $g \in L_{0}^{2}(\Gamma)$ and $\omega \in H\left(\operatorname{div}_{0}, \Omega\right)$, find $\mathbf{u} \in H^{1}(\Omega)^{3}$ satisfying

$$
\begin{array}{ll}
\operatorname{curl} \mathbf{u}=\omega & \text { in } \Omega, \\
\operatorname{div} \mathbf{u}=0 & \text { in } \Omega, \\
\mathbf{u} \cdot \mathbf{n}_{\Gamma}=g & \text { on } \Gamma
\end{array}
$$

If $\Gamma$ is smooth, the continuous problem can be easily analyzed, but the finite elements to discretize it are quite complicated $[19,10]$. If $\Gamma$ is polyhedric, then there are specific difficulties in studying the continuous problem [2, 7], but the finite elements are classical. We recall the main results of regularity for the solution of (7); these results depend on the regularity of the domain $\Omega$. The first result is proven in $[2,15]$.

Proposition 2.1. Assume that the bounded domain $\Omega$ is of class $\mathcal{C}^{1,1}$ or a convex polyhedron. Then we have the following continuous embedding:

$$
\left\{\mathbf{v} \in L^{2}(\Omega)^{3} \mid \mathbf{c u r l} \mathbf{v} \in L^{2}(\Omega)^{3}, \operatorname{div} \mathbf{v} \in L^{2}(\Omega), \mathbf{v} \cdot \mathbf{n}_{\Gamma} \in H^{1 / 2}(\Gamma)\right\} \hookrightarrow H^{1}(\Omega)^{3} ;
$$

as a consequence, problem (7) has a unique solution, in the sense of distributions, that belongs to $H^{1}(\Omega)^{3}$.

The solution $\mathbf{u}$ of problem (7) is computed as a sum of two functions that are solutions of two simpler problems, i.e., $\mathbf{u}=\mathbf{u}_{0}+\hat{\mathbf{u}}$, where $\hat{\mathbf{u}}$ is a divergence-free lifting in $\bar{\Omega}$ of a function $\hat{\mathbf{u}}_{\Gamma}$ defined on $\Gamma$ such that

$$
\hat{\mathbf{u}}_{\Gamma} \cdot \mathbf{n}_{\Gamma}=g, \quad \operatorname{div}_{\Gamma} \hat{\mathbf{u}}_{\Gamma}=0,
$$

and $\mathbf{u}_{0}$ satisfies

$$
\begin{array}{ll}
\operatorname{curl} \mathbf{u}_{0}=\omega-\operatorname{curl} \hat{\mathbf{u}} & \text { in } \Omega, \\
\operatorname{div} \mathbf{u}_{0}=0 & \text { in } \Omega, \\
\mathbf{u}_{0} \cdot \mathbf{n}_{\Gamma}=0 & \text { on } \Gamma .
\end{array}
$$


Note that, thanks to the introduction of a vector potential, (2) is exactly verified, whereas (1) is satisfied in the sense of distributions. Thanks to a trace result proved in [5], problem (7) is well-posed even in nonconvex polyhedra of $\mathbb{R}^{3}$ (such as a discretized torus).

Proposition 2.2. Let $\Gamma_{i}, i=1, \ldots, L$, be the faces of the boundary $\Gamma$ of a bounded polyhedron $\Omega$. There exists a real number $s>1 / 2$ such that for any function $g \in H^{1 / 2}\left(\partial \Gamma_{i}\right), i=1, \ldots, L$, problem (8) has a unique solution $\hat{\mathbf{u}} \in H^{s}(\Omega)^{3}$. In addition, for any $\omega \in H\left(\operatorname{div}_{0}, \Omega\right)$, problem (9) has a unique solution $\mathbf{u}_{0} \in H^{s}(\Omega)^{3}$.

We end this section by recalling and applying general results on vector fields defined on a regular bounded domain $\Omega$ of $\mathbb{R}^{3}$. We refer to $[2,3]$ and to their included references for the results. Let us introduce the following spaces:

$$
\begin{aligned}
& X_{T}(\Omega)=\left\{\mathbf{v} \in L^{2}(\Omega)^{3} \mid \operatorname{div} \mathbf{v} \in L^{2}(\Omega), \mathbf{c u r l} \mathbf{v} \in L^{2}(\Omega)^{3}, \mathbf{v} \cdot \mathbf{n}_{\Gamma} \in H^{1 / 2}(\Gamma)\right\}, \\
& X_{N}(\Omega)=\left\{\mathbf{v} \in L^{2}(\Omega)^{3} \mid \operatorname{div} \mathbf{v} \in L^{2}(\Omega), \mathbf{c u r l} \mathbf{v} \in L^{2}(\Omega)^{3}, \mathbf{v} \times \mathbf{n}_{\Gamma} \in H^{1 / 2}(\Gamma)^{3}\right\}, \\
& H_{T}(\Omega)=\left\{\mathbf{v} \in L^{2}(\Omega)^{3} \mid \operatorname{div} \mathbf{v}=0, \mathbf{c u r l} \mathbf{v}=\mathbf{0}, \mathbf{v} \cdot \mathbf{n}_{\Gamma}=0 \text { on } \Gamma\right\}, \\
& H_{N}(\Omega)=\left\{\mathbf{v} \in L^{2}(\Omega)^{3} \mid \operatorname{div} \mathbf{v}=0, \mathbf{c u r l} \mathbf{v}=\mathbf{0}, \mathbf{v} \times \mathbf{n}_{\Gamma}=\mathbf{0} \text { on } \Gamma\right\}, \\
& P_{T}: X_{T}(\Omega) \rightarrow H_{T}(\Omega) \text { orthogonal projection operator, } \\
& P_{N}: X_{N}(\Omega) \rightarrow H_{N}(\Omega) \text { orthogonal projection operator, } \\
& W^{1}(\Omega)=\left\{\mathbf{w} \in H^{1}(\Omega)^{3} \mid \operatorname{div} \mathbf{w}=0, \mathbf{w} \times \mathbf{n}_{\Gamma}=\mathbf{0}, \int_{\Gamma} \mathbf{w} \cdot \mathbf{n}_{\Gamma} d \Gamma=0\right\}
\end{aligned}
$$

Theorem 2.3 (Hodge decomposition). For a given $\mathbf{u} \in L^{2}(\Omega)^{3}$, we have two possible decompositions:

$$
\mathbf{u}=\operatorname{grad} \phi+\operatorname{curl} \mathbf{w}+\theta
$$

with $\theta \in H_{T}(\Omega)$ and a unique $(\phi, \mathbf{w})$ verifying $\phi \in H^{1}(\Omega) \cap L_{0}^{2}(\Omega)$, w $\in W^{1}(\Omega)$;

$$
\text { (ii) } \quad \mathbf{u}=\operatorname{grad} \psi+\operatorname{curl} \mathbf{p}+\eta
$$

with $\eta \in H_{N}(\Omega)$ and a unique $(\psi, \mathbf{p})$ verifying $\psi \in H_{0}^{1}(\Omega)$ and

$$
\mathbf{p} \in H^{1}(\Omega)^{3}, \operatorname{div} \mathbf{p}=0, \mathbf{p} \cdot \mathbf{n}_{\Gamma}=0, P_{T} \mathbf{p}=\mathbf{0} .
$$

The decomposition (i) (resp., (ii)) of a field $\mathbf{u}$ is into three orthogonal components of the type $\operatorname{grad} \phi$ (resp., $\operatorname{grad} \psi$ ) plus curlw (resp., $\operatorname{curl} \mathbf{p}$ ) plus a vector lying in $H_{T}(\Omega)\left(\right.$ resp., $H_{N}(\Omega)$ ).

Theorem 2.4 (Foias, Temam [12]). Let $\mathbf{u} \in L^{2}(\Omega)^{3}$ and $\mathbf{p} \in H(\mathbf{c u r l}, \Omega)$ such that $\mathbf{u}=\mathbf{c u r l} \mathbf{p}$; then $P_{N} \mathbf{u}=\mathbf{0}$.

If a vector $\mathbf{u}$ admits a representation in terms of a vector potential $\mathbf{p}$, i.e., $\mathbf{u}=$ $\operatorname{curl} \mathbf{p}$, it clearly satisfies the condition $\operatorname{div} \mathbf{u}=0$. Moreover, for any vector $\mathbf{u}$ of the form (ii), Theorem 2.4 yields $P_{N} \mathbf{u}=\mathbf{0}$, a condition which precludes flow problems with sources and sinks, as remarked in [10]. Finally, since the scalar $\psi \in H_{0}^{1}(\Omega)$, we have $\psi=0$. The vector potential $\mathbf{p}$ is then the right tool to represent the field $\mathbf{u}$ solution of the considered problem.

In the next section, we recall the main properties of the finite elements we are going to use to discretize $\mathbf{p}$. These finite elements are $H(\mathbf{c u r l}, \Omega)$-conforming, and by consequence, the field $\mathbf{u}$ will be approximated by $H(\operatorname{div}, \Omega)$-conforming finite elements. Throughout the paper, we treat the three-dimensional case. 


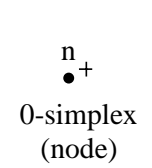

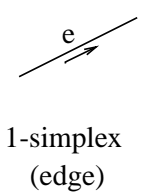

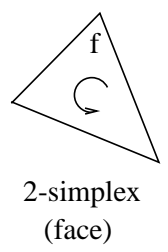

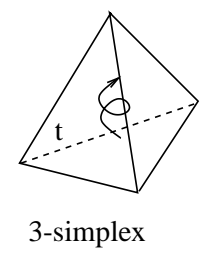

(volume)

Fig. 1. Example of oriented p-simplex, $p=0, \ldots, 3$.

3. Meshing the domain with cellular complexes. Given a domain $\Omega \subset \mathbb{R}^{3}$ with boundary $\Gamma$, a simplicial mesh $m$ in $\Omega$ is a tessellation of $\bar{\Omega}$ by tetrahedra, under the condition that any two of them may intersect along a common face, edge, or node but in no other way. We denote by $\mathcal{N}_{m}, \mathcal{E}_{m}, \mathcal{F}_{m}$, and $\mathcal{T}_{m}$ (nodes, edges, faces, and tetrahedra, respectively) the sets of simplices of dimension 0 to 3 thus obtained, and by $N_{m}, E_{m}, F_{m}$, and $T_{m}$ their cardinalities. The importance of simplicial meshes lies in the fact that any triangulated domain is homeomorphic to one in which the triangles are flat and the edges straight. Properties on the mesh will hold for the domain, as we are going to present in the following.

First we need to underline some combinatorial properties of the simplicial mesh. Let $\mathcal{M}(r, s)$ denote the set of matrices $A$ whose elements are $A(i, j)$ with $1 \leq i \leq r$ and $1 \leq j \leq s$. In addition to the list of nodes and their positions, the mesh data structure also contains incidence matrices, saying which node belongs to which edge, which edge bounds which face, etc. [6, 14]. There is a notion of orientation for the simplex as in Figure 1 that has to be taken into account to define the incidence matrices. In short, an edge is not only a two-node subset of $\mathcal{N}_{m}$ but an ordered such set, where the order implies an orientation. Let $e=\{\ell, n\}$ be an edge of the mesh oriented from the node $\ell$ to $n$. We can define the incidence numbers $G_{e, n}=1$, $G_{e, \ell}=-1$, and $G_{e, k}=0$ for all nodes $k$ other than $\ell$ and $n$. These numbers form a rectangular matrix $G \in \mathcal{M}\left(E_{m}, N_{m}\right)$, which describes how edges connect to nodes. A face $f=\{\ell, n, k\}$ has three vertices which are the nodes $\ell, n, k$. Note that $\{n, k, l\}$ and $\{k, l, n\}$ denote the same face $f$, whereas $\{n, l, k\}$ denotes an oppositely oriented face, which is not supposed to belong to $\mathcal{F}_{m}$ if $f$ does. An orientation of $f$ induces an orientation of its boundary. So, with respect to the orientation of the face $f$, the one of the edge $\{l, n\}$ is positive, and the one of $\{k, n\}$ is negative. Then we can define the incidence number $R_{f, e}=1$ (resp., -1 ) if the orientation of $e$ matches (resp., does not match) the one on the boundary of $f$, and $R_{f, e}=0$ if $e$ is not an edge of $f$. These numbers form a matrix $R \in \mathcal{M}\left(F_{m}, E_{m}\right)$. Finally, let us consider the tetrahedron $t=\{k, l, m, n\}$ positively oriented if the three vectors $\{k, l\},\{k, m\}$, and $\{k, n\}$ define a positive frame. $\left(t^{\prime}=\{l, m, n, k\}\right.$ has a negative orientation and does not belong to $\mathcal{T}_{m}$ if $t$ does.) A third matrix $D \in \mathcal{M}\left(T_{m}, F_{m}\right)$ can be defined by setting $D_{t, f}= \pm 1$ if face $f$ bounds the tetrahedron $t$, with the sign depending on whether the orientation of $f$ and of the boundary of $t$ match or not, and $D_{t, f}=0$ in case $f$ does not bound $t$. For consistency, we attribute an orientation to nodes as well. Implicitly, we have been orienting all nodes the same way $(+1)$ up to now. Note that a sign $(-1)$ to node $n$ changes the sign of all entries of column $n$ in the above $G$. It can be easily proven that $R G=0$ and $D R=0[6]$.

We now define the mixed finite elements we use $[6,23,24,26]$ : they are scalar functions or vector fields associated to all the simplices of the mesh $m$. We start by 
denoting $\varphi_{n}$ the only continuous, piecewise affine function, which is equal to 1 at $n$ and to 0 at other nodes. We set $W_{m}^{0}=\operatorname{span}\left\{\varphi_{n} \mid n \in \mathcal{N}_{m}\right\}$. The degree (zero in this case) of the elements of $W_{m}^{0}$ refers to the dimension of the simplices they are associated with (i.e., nodes) and not to the degree of $\varphi_{n}$ as a polynomial. To the edge $e$, let us associate the vector field $\mathbf{w}_{e}$ of the form $\mathbf{a} \times \mathbf{x}+\mathbf{b}$ in each tetrahedron $t \in \mathcal{T}_{m}$; the two vectors $\mathbf{a}$ and $\mathbf{b}$ are determined by imposing that the circulation of $\mathbf{w}_{e}$ along $e \in t$ is 1 and 0 along the other edges of $t$. We denote $W_{m}^{1}=\operatorname{span}\left\{\mathbf{w}_{e} \mid e \in \mathcal{E}_{m}\right\}$. Similarly, $W_{m}^{2}=\operatorname{span}\left\{\mathbf{v}_{f} \mid f \in \mathcal{F}_{m}\right\}$ with $\mathbf{v}_{f}$ the vector of the form $a \mathbf{x}+\mathbf{b}$ in each tetrahedron $t \in \mathcal{T}_{m}$; the scalar $a$ and the vector $\mathbf{b}$ are determined by imposing that the flux of $\mathbf{v}_{f}$ across the face $f \in t$ is 1 and 0 across the other faces of $t$. Finally, we introduce $W_{m}^{3}=\operatorname{span}\left\{\mu_{t} \mid t \in \mathcal{T}_{m}\right\}$, where $\mu_{t}$ is the only scalar whose integral over $t$ is 1 and 0 over the other tetrahedra. ${ }^{1}$

Note that, given two adjacent tetrahedra $t$ and $t^{\prime}$ sharing a face $f$, the function $\varphi_{n}$ and both the tangential component of $\mathbf{w}_{e}$ and the normal component of $\mathbf{v}_{f}$ are continuous across $f$, whereas the function $\mu_{t}$ is not. Thanks to these continuity properties, $W_{m}^{0} \subset H^{1}(\Omega), W_{m}^{1} \subset H(\operatorname{curl}, \Omega), W_{m}^{2} \subset H(\operatorname{div}, \Omega)$, and $W_{m}^{3} \subset L^{2}(\Omega)$. The spaces $W_{m}^{p}, p=0,1,2,3$, have finite dimension given by $N_{m}, E_{m}, F_{m}, T_{m}$, respectively, and they play the role of Galerkin approximation spaces for the latter functional spaces.

The properties introduced so far concern the spaces $W_{m}^{p}$ taken one by one. There are properties of the structure made of the spaces $W_{m}^{p}$ when taken together. We know that the following inclusions hold:

$$
\operatorname{grad} W_{m}^{0} \subset W_{m}^{1}, \quad \operatorname{curl} W_{m}^{1} \subset W_{m}^{2}, \quad \operatorname{div} W_{m}^{2} \subset W_{m}^{3} .
$$

It is natural to ask when the sequence

$$
\{0\} \longrightarrow W_{m}^{0} \stackrel{\text { grad }}{\longrightarrow} W_{m}^{1} \stackrel{\text { curl }}{\longrightarrow} W_{m}^{2} \stackrel{\text { div }}{\longrightarrow} W_{m}^{3} \longrightarrow\{0\}
$$

is exact at levels 1 and 2, i.e., when it happens that

$$
\operatorname{ker}\left(\operatorname{curl} ; W_{m}^{1}\right)=\operatorname{grad} W_{m}^{0}, \quad \operatorname{ker}\left(\operatorname{div} ; W_{m}^{2}\right)=\operatorname{curl} W_{m}^{1},
$$

where

$$
\operatorname{ker}\left(\operatorname{curl} ; W_{m}^{1}\right):=W_{m}^{1} \cap \operatorname{ker}(\operatorname{curl}), \quad \operatorname{ker}\left(\operatorname{div} ; W_{m}^{2}\right):=W_{m}^{2} \cap \operatorname{ker}(\operatorname{div}) .
$$

At levels 0 and 3 , we lose the property of exactitude for the previous sequence because, at level 0 , the gradient operator is not injective, and, at level 3 , the divergence operator is not surjective. The Poincaré lemma states that, when the domain $\Omega$ is contractible [14], the image fills the kernel in both cases. This is not the case with $\Omega$ nonsimply connected; for example, we have in fact that $\operatorname{grad}\left(W_{m}^{0}\right)$ is a proper subset of $\operatorname{ker}\left(\operatorname{curl} ; W_{m}^{1}\right)$. This tells us something about the topology of $\Omega$; namely, the

\footnotetext{
${ }^{1}$ Given the nodes $n, l, m, k$, the edge $e=\{l, m\}$, the face $f=\{l, m, k\}$, and the tetrahedron $t=\{i, j, k, l\}$, the generators of the spaces $W_{m}^{p}, p=0,1,2,3$, respectively, can also be defined as follows $\left(\lambda_{n}\right.$ is the barycentric coordinate associated to $\left.n\right)$ :

$$
\begin{aligned}
\varphi_{n}= & \lambda_{n}, \quad \mathbf{w}_{e}=\lambda_{l} \operatorname{grad} \lambda_{m}-\lambda_{m} \operatorname{grad} \lambda_{l}, \\
\mathbf{v}_{f}=2 & \left(\lambda_{l} \operatorname{grad} \lambda_{m} \times \operatorname{grad} \lambda_{k}+\lambda_{m} \operatorname{grad} \lambda_{k} \times \operatorname{grad} \lambda_{l}+\lambda_{k} \operatorname{grad} \lambda_{l} \times \operatorname{grad} \lambda_{m}\right), \\
\mu_{t}=6 & \left(\lambda_{i} \operatorname{grad} \lambda_{j} \times \operatorname{grad} \lambda_{k} \cdot \operatorname{grad} \lambda_{l}+\lambda_{j} \operatorname{grad} \lambda_{k} \times \operatorname{grad} \lambda_{l} \cdot \operatorname{grad} \lambda_{i}\right. \\
& \left.\quad+\lambda_{k} \operatorname{grad} \lambda_{l} \times \operatorname{grad} \lambda_{i} \cdot \operatorname{grad} \lambda_{j}+\lambda_{l} \operatorname{grad} \lambda_{i} \times \operatorname{grad} \lambda_{j} \cdot \operatorname{grad} \lambda_{k}\right)=[\operatorname{vol}(t)]^{-1} .
\end{aligned}
$$
}


presence of $b_{1}$ "loops," where $b_{1}=\operatorname{dim}\left[\operatorname{ker}\left(\mathbf{c u r l} ; W_{m}^{1}\right) / \operatorname{grad}\left(W_{m}^{0}\right)\right]$ is the Betti number of dimension 1 of the domain. Solenoidal fields which are not curls indicate the presence of $b_{2}$ "holes," where $b_{2}=\operatorname{dim}\left[\operatorname{ker}\left(\operatorname{div} ; W_{m}^{2}\right) / \operatorname{curl}\left(W_{m}^{1}\right)\right]$ is the Betti number of dimension 2 of the domain. These are global topological properties of the meshed domain; they do not depend on the mesh that is used to compute them, but they are intrinsic to the considered domain $\Omega$. The sequences are thus an algebraic tool by which the topology of $\Omega$ can be explored (and this was Whitney's concern [31]).

The connection between the vector field picture and the cohomological picture in the electromagnetic context has also been considered more recently in $[22,30]$.

4. Chains, boundary homomorphism, and homology groups. Let $m$ be the simplicial mesh on $\Omega \subset \mathbb{R}^{3}$. A $p$-chain $c$ is an assignement to each simplex of dimension $p$ in $m$ of a number $\alpha$, and we denote by $C_{p}(m)$ the set of all $p$-chains. The set $C_{p}(m)$ has a structure of an abelian group with respect to the addition of $p$-chains; two $p$-chains are added by adding the corresponding coefficients.

To give an example, let us consider a path of edges of the mesh $m$ to go from a point $n_{1}$ to a point $n_{2}$; it is an oriented line. Assigning an integer $\alpha_{e}$ equal to +1 or -1 when the edge $e$ belongs to the path and its orientation is in agreement or in disagreement with that of the path and 0 for all edges $e$ that do not belong to the path, we define a 1-chain. A circuit is a line plus a way to run along it; so, when the line is made of oriented edges, we need to tell the positive direction along each edge, which is precisely what the chain coefficient $\alpha_{e}$ does. We remark that "chain" is a more general concept than "path," "circuit," etc. In our case, we assume that all coefficients $\alpha_{i}$ are relative integers.

The next concept is the boundary operator $\partial_{p}: C_{p}(m) \rightarrow C_{p-1}(m), p>0$. By definition, we have

$$
\partial_{1}(e)=\sum_{n \in \mathcal{N}_{m}} G_{e, n} n, \quad \partial_{2}(f)=\sum_{e \in \mathcal{E}_{m}} R_{f, e} e, \quad \partial_{3}(t)=\sum_{f \in \mathcal{F}_{m}} D_{t, f} f .
$$

Note that $\partial_{p}$ is represented by a matrix that is $G^{t}, R^{t}$, or $D^{t}$ depending on the dimension $p>0$. We remark, in particular, that $\partial_{p+1} \circ \partial_{p}=0$, i.e. the boundary of a boundary is the zero chain.

We will say that a $p$-chain $c$ is closed if $\partial_{p} c=0$. Nontrivial closed $p$-chains are called $p$-cycles and constitute the subspace $Z_{p}(m)=\operatorname{ker}\left(\partial_{p} ; C_{p}(m)\right)$. A $p$-chain $c$ is a boundary if there is a $(p+1)$-chain $\gamma$ such that $c=\partial_{p+1} \gamma$. The $p$-boundaries constitute the subspace $B_{p}(m)=\partial_{p+1} C_{p+1}(m)$. Both $Z_{p}(m)$ and $B_{p}(m)$ are abelian groups with respect to the addition of $p$-chains. Boundaries are cycles, but not all cycles are boundaries; we have in fact that $B_{p}(m) \subset Z_{p}(m)$.

The quotient space $H_{p}(m)=\left[Z_{p}(m) / B_{p}(m)\right]$ is the homology group of order $p$ of the mesh $m$, and the Betti number $b_{p}$ is equal to $\operatorname{dim}\left[H_{p}(m)\right]$. In particular, we have that $b_{0}=\operatorname{dim}\left[\operatorname{ker}\left(\operatorname{grad} ; W_{m}^{0}\right)\right]$ is the number of connected components of $\Omega$, and $b_{3}=\operatorname{dim}\left[\operatorname{div}\left(W_{m}^{3}\right)\right]$ is the number of connected components of $\Gamma$ minus one.

Our concern is to determine the cycles that are not boundaries for $p=1$ and 2, i.e., to computate the generators of $H_{1}(m)$ and $H_{2}(m)$. Triangulating a domain reduces the calculation of $H_{p}(m)$ to a finite procedure (in section 8, we present an algebraic algorithm to define a basis of $H_{p}(m), p=1$ and 2); the remarkable thing is that homology groups, in spite of being defined via triangulation, do measure something intrinsic and geometrical (they are topological invariants; i.e., they depend on the domain up to a homeomorphism) that does not depend on the mesh. The homology groups of a surface have a direct link with the possibility of representing curl-free 
(resp., divergence-free) vectors as gradients (resp., curls). This link is a determinant in the construction of numerical algorithms for solving given problems in terms of scalar or vector potentials, as we are going to see. A key tool in this construction is the Euler-Poincaré characteristic of $\Omega$ and its boundary $\Gamma$ [14].

Remark 4.1. Given a connected domain $\Omega$, the Euler-Poincaré characteristic of $\Omega$ is the integer

$$
\chi(\Omega)=N_{m}-E_{m}+F_{m}-T_{m},
$$

where $N_{m}, E_{m}, F_{m}$, and $T_{m}$ denote, respectively, the number of nodes, edges, faces, and tetrahedra of the mesh $m$ discretizing $\bar{\Omega}$.

Remark 4.2. Given a connected orientable surface $\Gamma$, the Euler-Poincaré characteristic of $\Gamma$ is the integer

$$
\chi(\Gamma)=N_{m}^{\Gamma}-E_{m}^{\Gamma}+F_{m}^{\Gamma},
$$

where $N_{m}^{\Gamma}, E_{m}^{\Gamma}$, and $F_{m}^{\Gamma}$ denote, respectively, the number of nodes, edges, and triangles of the mesh $m^{\Gamma}$ discretizing $\bar{\Gamma}$.

The Euler-Poincaré characteristic is linked to the homology groups' dimension as follows:

$$
\chi(\Gamma)=b_{0}^{\Gamma}-b_{1}^{\Gamma}+b_{2}^{\Gamma}, \quad \chi(\Omega)=b_{0}-b_{1}+b_{2}-b_{3},
$$

where $b_{i}^{\Gamma}, i=0,1,2$ (resp., $b_{i}, i=0,1,2,3$ ), are the Betti numbers of $\Gamma$ (resp., $\Omega$ ). The major point is that these numbers, and consequently the Euler-Poincaré characteristic, are topological invariants. For more details on the subject, see [29].

5. Some discrete spaces and tools. Let us consider a triangulation $m$ of $\bar{\Omega}$, its restriction $m^{\Gamma}$ to the boundary $\Gamma$ of $\Omega$. Let us define the following two functional spaces on $\Omega$ and their analogues on the boundary $\Gamma$ :

$$
\begin{array}{ll}
W_{m, 0}^{2}=\left\{\mathbf{v} \in W_{m}^{2} \mid \mathbf{v} \cdot \mathbf{n}_{\Gamma}=0 \text { on } \Gamma\right\}, & W_{m^{\Gamma}}^{2}=\left\{\pi \mathbf{v} \mid \mathbf{v} \in W_{m}^{2}\right\}, \\
W_{m, 0}^{1}=\left\{\mathbf{v} \in W_{m}^{1} \mid \mathbf{v} \times \mathbf{n}_{\Gamma}=\mathbf{0} \text { on } \Gamma\right\}, & W_{m^{\Gamma}}^{1}=\left\{\pi \mathbf{v} \mid \mathbf{v} \in W_{m}^{1}\right\} .
\end{array}
$$

Note that $W_{m^{\Gamma}}^{1}$ is the restriction to $\Gamma$ of the space $W_{m}^{1}$ in the sense that its vectors are associated to the mesh edges belonging to $m^{\Gamma}$. Similarly, the space $W_{m^{\Gamma}}^{2}$, also known as the Raviart-Thomas element space, is the restriction to $\Gamma$ of $W_{m}^{2}$. Its vectors $\mathbf{v}_{e}$ are tangential to $\Gamma$ and, in each triangle $f \in m^{\Gamma}$, are determined by imposing that the flux of $\mathbf{v}_{e}$ across the edge $e \in f$ is 1 and 0 across the other edges of $f$. Vectors of the space $W_{m^{\Gamma}}^{2}$ are adapted to represent flux densities that are tangential to $\Gamma$.

We look for a discrete approximation $\mathbf{u}_{m}$ of $\mathbf{u}$ on the mesh $m$ of the form

$$
\mathbf{u}_{m}=\operatorname{curl} \mathbf{p}_{m}
$$

with $\mathbf{u}_{m}$ (resp., $\mathbf{p}_{m}$ ) lying in $W_{m}^{2}$ (resp., $W_{m}^{1}$ ). The uniqueness of the potential $\mathbf{p}_{m}$ is automatically satisfied if we choose $\mathbf{p}_{m} \in W_{m}^{1}$ and we add a gauge condition. In the following, a linear space for the discrete potential $\mathbf{p}_{m}$ is proposed; we treat the gauge condition in an entirely algebraic way and obtain the so-called axial gauge [16].

To define the discrete space of the vector potential, we need some details on the graph defined in the set of vertices of $m$ by the mesh edges. For a general reference on graph theory, we suggest [14]. 


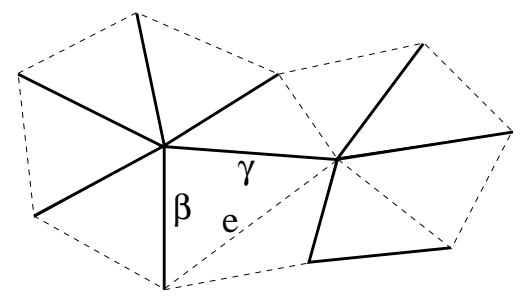

FIG. 2. In the given mesh $m$, the thick dark edges compose the spanning tree $\mathcal{T}$, and the dashed ones compose the corresponding cotree $\mathcal{E}_{m} \backslash \mathcal{T}$. The coedge e closes a circuit together with the tree edges $\beta$ and $\gamma$.

A set $\mathcal{T}$ of edges of the mesh $m$ such that $C_{1}(\mathcal{T})$ does not contain any cycle is called a tree. A tree is a spanning tree if there is no strictly larger tree containing it. The set of all left-over edges, i.e., $\mathcal{E}_{m} \backslash \mathcal{T}$, is called the associated cotree, and its elements are the coedges with respect to $\mathcal{T}$. Coedges thus furnish a basis for 1-cycles in the sense that, given a coedge $e$, there is a unique way to assign an integer $\alpha_{\epsilon}$ to each edge $\epsilon$ of the tree in order to get a closed 1-chain: $\partial\left(e+\sum_{\epsilon \in \mathcal{T}} \alpha_{\epsilon} \epsilon\right)=0$. In short, one says that each coedge $e \in \mathcal{E}_{m} \backslash \mathcal{T}$ "closes a circuit" $C_{e}$ in conjunction with edges of the tree. (An example is given in Figure 2, where $C_{e}=\{e\} \cup\{\beta\} \cup\{\gamma\}$.)

Remark 5.1. For a given mesh $m$ of the domain $\Omega$, the number of edges contained in a spanning tree $\mathcal{T}$ can be expressed in terms of the Betti numbers of the domain by means of the following formula (with easy recursive proof):

$$
\# \mathcal{T}=b_{1}+\left(N_{m}-b_{0}\right)
$$

For contractible domains, we have $\# \mathcal{T}=N_{m}-1$. For noncontractible ones, the spanning tree is enriched with additional edges to take into account that there are 1 -cycles that do not bound a surface $\left(b_{1} \neq 0\right)$. (The enriched spanning tree has been called a "belted spanning tree" in [6].)

Now, we explain how to use trees and cotrees to define the proper approximation space to solve the considered problem. In the following sections, $\mathcal{T}$ (resp., $\mathcal{T}^{\Gamma}$ ) always represents a spanning tree on $\Omega$ (resp., $\Gamma$ ).

6. Approximation of the problem in the simply connected case. We are interested here in solving problem (7); the domain $\Omega$ and its boundary $\Gamma$ are assumed to be connected and simply connected. We thus assume that $\Omega$ is a sphere (up to a homeomorphism). The nonsimply connected case is addressed in the next section.

6.1. Lifting the boundary condition for a sphere. As in [10], given $\mathcal{T}^{\Gamma}$ and $g \in L_{0}^{2}(\Gamma)$, we construct a vector $\hat{\mathbf{u}}_{m}^{\Gamma}$ in $W_{m^{\Gamma}}^{2} \operatorname{such}$ that $\operatorname{div}_{\Gamma} \hat{\mathbf{u}}_{m}^{\Gamma}=0, \hat{\mathbf{u}}_{m}^{\Gamma} \cdot \mathbf{n}_{\Gamma}=g$ face by face on $\Gamma$, we show that $\hat{\mathbf{u}}_{m}^{\Gamma}$ is unique, and we define $\hat{\mathbf{u}} \in W_{m}^{2}$ as the divergence-free lifting of $\hat{\mathbf{u}}_{m}^{\Gamma}$ in $\Omega$. Problem (8) is thus well-posed.

Proposition 6.1. Let us consider a triangulation $m$ of $\bar{\Omega}$, its restriction $m^{\Gamma}$ to the boundary $\Gamma$ of $\Omega$, and a spanning tree $\mathcal{T}^{\Gamma}$ in $m^{\Gamma}$. Let $\Gamma$ be a sphere and $g \in L_{0}^{2}(\Gamma)$. There is a unique divergence-free vector $\hat{\mathbf{u}}_{m}^{\Gamma} \in W_{m^{\Gamma}}^{2}$ of the form

$$
\hat{\mathbf{u}}_{m}^{\Gamma}=\sum_{e \in \mathcal{E}_{m}^{\Gamma} \backslash \mathcal{T}^{\Gamma}} \hat{u}_{e} \operatorname{curl} \mathbf{w}_{e}, \quad \mathbf{w}_{e} \in W_{m^{\Gamma}}^{1}
$$




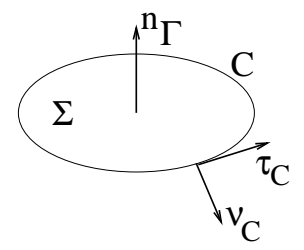

FIG. 3. Due to the simple connectedness of $\Gamma$, any circuit $C$ in $m^{\Gamma}$ bounds a surface $\Sigma \subset \Gamma$.

that satisfies

$$
\int_{f} \hat{\mathbf{u}}_{m}^{\Gamma} \cdot \mathbf{n}_{\Gamma}=\int_{f} g \quad \forall f \in \mathcal{F}_{m}^{\Gamma}
$$

Proof. Let us introduce the two spaces $\operatorname{ker}\left(\operatorname{div}_{\Gamma} ; W_{m^{\Gamma}}^{2}\right)$ and

$$
\mathcal{V}\left(\mathcal{T}^{\Gamma}\right)=\operatorname{span}\left\{\mathbf{w}_{e} \mid \mathbf{w}_{e} \in W_{m^{\Gamma}}^{1}, e \in \mathcal{E}_{m}^{\Gamma} \backslash \mathcal{T}^{\Gamma}\right\}
$$

The curl operator is well defined as $\mathcal{V}\left(\mathcal{T}^{\Gamma}\right) \rightarrow \operatorname{ker}\left(\operatorname{div}_{\Gamma} ; W_{m^{\Gamma}}^{2}\right)$.

The curl mapping $\mathcal{V}\left(\mathcal{T}^{\Gamma}\right) \rightarrow \operatorname{ker}\left(\operatorname{div}_{\Gamma} ; W_{m^{\Gamma}}^{2}\right)$ is injective. Let us consider the vector $\hat{\mathbf{p}}_{m}^{\Gamma} \in \mathcal{V}\left(\mathcal{T}^{\Gamma}\right)$ of the form

$$
\hat{\mathbf{p}}_{m}^{\Gamma}=\sum_{e \in \mathcal{E}_{m}^{\Gamma} \backslash \mathcal{T}^{\Gamma}} \hat{u}_{e} \mathbf{w}_{e}
$$

Let $\alpha \in \mathcal{E}_{m}^{\Gamma} \backslash \mathcal{T}^{\Gamma}$ be a given coedge, and let $C \subset\{\alpha\} \cup \mathcal{T}^{\Gamma}$ be the associated cycle; then we have

$$
\int_{C} \hat{\mathbf{p}}_{m}^{\Gamma} \cdot \tau_{C}=\hat{u}_{\alpha} \int_{\alpha} \mathbf{w}_{\alpha} \cdot \tau_{C}=\hat{u}_{\alpha}
$$

where $\tau_{C}$ is the tangential vector to $C$.

On the other hand, since $\Gamma$ is simply connected, $C$ is the boundary of a surface $\Sigma$ contained in $\Gamma$. On $\Gamma$, the normal $\nu_{C}$ and tangential $\tau_{C}$ vectors to $C$ are linked to $\mathbf{n}_{\Gamma}$ through the relation $\nu_{C} \times \tau_{C}=\mathbf{n}_{\Gamma}$ (see Figure 3). By definition, we have that

$$
\left(\operatorname{curl} \hat{\mathbf{p}}_{m}^{\Gamma}\right) \cdot \mathbf{n}_{\Gamma}=\operatorname{curl}_{\Gamma}\left(\pi \hat{\mathbf{p}}_{m}^{\Gamma}\right)=\operatorname{div}_{\Gamma}\left(\hat{\mathbf{p}}_{m}^{\Gamma} \times \mathbf{n}_{\Gamma}\right)
$$

The Stokes theorem and the previous tools yield

$$
\hat{u}_{\alpha}=\int_{C} \hat{\mathbf{p}}_{m}^{\Gamma} \cdot \tau_{C}=\int_{C}\left(\hat{\mathbf{p}}_{m}^{\Gamma} \times \mathbf{n}_{\Gamma}\right) \cdot \nu_{C}=\int_{\Sigma} \operatorname{div}_{\Gamma}\left(\hat{\mathbf{p}}_{m}^{\Gamma} \times \mathbf{n}_{\Gamma}\right)=\int_{\Sigma}\left(\operatorname{curl} \hat{\mathbf{p}}_{m}^{\Gamma}\right) \cdot \mathbf{n}_{\Gamma} .
$$

If curl $\hat{\mathbf{p}}_{m}^{\Gamma}=\mathbf{0}$, then $\hat{u}_{\alpha}=0$ for all $\alpha \in \mathcal{E}_{m}^{\Gamma} \backslash \mathcal{T} \mathcal{T}^{\Gamma}$, yielding $\hat{\mathbf{p}}_{m}^{\Gamma}=\mathbf{0}$.

Let $W$ be the space composed of vectors in $\operatorname{ker}\left(\operatorname{div}_{\Gamma} ; W_{m^{\Gamma}}^{2}\right)$ verifying (15) with $g \in L_{0}^{2}(\Gamma)$. The linear spaces $\mathcal{V}\left(\mathcal{T}^{\Gamma}\right)$ and $W$ have the same dimension. On one hand, we have $\operatorname{dim}\left[\mathcal{V}\left(\mathcal{T}^{\Gamma}\right)\right]=E_{m}^{\Gamma}-\left(N_{m}^{\Gamma}-1\right)$ thanks to Remark 5.1, and on the other hand, $\operatorname{dim}[W]=F_{m}^{\Gamma}-1$. Due to the fact that the Euler-Poincaré characteristic for a spherical surface is 2 , Remark 4.2 yields

$$
E_{m}^{\Gamma}-N_{m}^{\Gamma}+1=F_{m}^{\Gamma}-1
$$

Given a spanning tree $\mathcal{T}^{\Gamma}$ and a scalar function $g \in L_{0}^{2}(\Gamma)$, there is a unique divergence-free vector of the form (14) and verifying (15). 


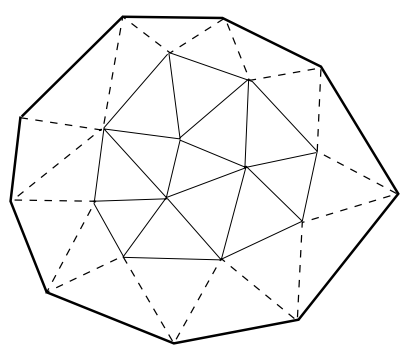

FIG. 4. In this two-dimensional example, the thick dark edges constitute the set $\mathcal{E}_{m}^{\Gamma}$, the dashed edges define $\mathcal{E}_{m}^{\ell}$, and the light ones compose $\mathcal{E}_{m}^{\text {int }}$. Note that here $\mathcal{E}_{m}^{B}$ is empty but it is not always the case with more general three-dimensional meshes $m$.

6.2. Interior problem for a sphere. At this point we can write

$$
\mathbf{u}_{m}=\hat{\mathbf{u}}_{m}+\sum_{e \in ?} u_{e} \operatorname{curl} \mathbf{w}_{e},
$$

where $\hat{\mathbf{u}}_{m}$ is the solution of problem (8) in the sense given in Proposition 6.1 and the symbol "?" in the previous sum is there on purpose to indicate that we do not know yet to which set of internal coedges we have to extend the sum. We remark that

$$
\mathcal{E}_{m}=\mathcal{E}_{m}^{\Gamma} \cup \mathcal{E}_{m}^{\mathrm{int}} \cup \mathcal{E}_{m}^{\ell} \cup \mathcal{E}_{m}^{B},
$$

where $\mathcal{E}_{m}^{\ell}$ is the set of mesh edges having only one extremity on $\Gamma, \mathcal{E}_{m}^{\text {int }}$ is the set of mesh edges having both extremities in $\Omega$, and $\mathcal{E}_{m}^{B}$ is the set of mesh edges interior to $\Omega$ but with both extremities on $\Gamma$ (see the example in Figure 4 ).

We denote $\mathcal{T}^{\text {int }}$ a spanning tree contained in $\mathcal{E}_{m}^{\text {int }}$ and $\mathcal{T}^{\ell}$ a subset of $\mathcal{E}_{m}^{\ell}$ composed of one edge since $\Gamma$ is connected, linking $\mathcal{T}^{\text {int }}$ to $\mathcal{T}^{\Gamma}$ and

$$
\mathcal{U}_{m}^{0}=\left(\mathcal{E}_{m}^{\text {int }} \cup \mathcal{E}_{m}^{\ell} \cup \mathcal{E}_{m}^{B}\right) \backslash\left(\mathcal{T}^{\text {int }} \cup \mathcal{T}^{\ell}\right) .
$$

In the next proposition, we prove that problem (9) is well-posed at the discrete level. In particular, given $\mathcal{T}^{\text {int }} \cup \mathcal{T}^{\ell}$ and a function $\omega \in \operatorname{ker}\left(\operatorname{div} ; W_{m}^{2}\right)$, we construct a divergence-free vector $\mathbf{u}_{m}^{0} \in W_{m}^{2}$ such that curl $\mathbf{u}_{m}^{0}=\omega-\operatorname{curl} \hat{\mathbf{u}}_{m}$ in $\Omega$ and $\mathbf{u}_{m}^{0} \cdot \mathbf{n}_{\Gamma}=0$ face by face on $\Gamma$, and we show that $\mathbf{u}_{m}^{0}$ is unique.

Proposition 6.2. Let us consider a triangulation $m$ of $\bar{\Omega}$ and a spanning tree $\mathcal{T}^{\text {int }} \cup \mathcal{T}^{\ell}$ in $m \backslash m^{\Gamma}$. Let us suppose that $\Omega$ is a sphere and $\omega \in \operatorname{ker}\left(\operatorname{div} ; W_{m}^{2}\right)$. There exists a unique divergence-free vector $\mathbf{u}_{m}^{0} \in W_{m, 0}^{2}$ of the form

$$
\mathbf{u}_{m}^{0}=\sum_{e \in \mathcal{U}_{m}^{0}} u_{e}^{0} \operatorname{curl} \mathbf{w}_{e}, \quad \mathbf{w}_{e} \in W_{m}^{1}
$$

that satisfies

$$
\operatorname{curl} \mathbf{u}_{m}^{0}=\omega-\operatorname{curl} \hat{\mathbf{u}}_{m} \quad \text { in } \Omega,
$$

where $\hat{\mathbf{u}}_{m}$ is the divergence-free lifting in $\Omega$ of $\hat{\mathbf{u}}_{m}^{\Gamma}$ defined in Proposition 6.1.

Proof. Let us introduce the two spaces $\operatorname{ker}\left(\operatorname{div} ; W_{m, 0}^{2}\right)$ and

$$
\mathcal{V}\left(\mathcal{T}^{\text {int }}, \mathcal{T}^{\ell}\right)=\operatorname{span}\left\{\mathbf{w}_{e} \mid \mathbf{w}_{e} \in W_{m}^{1}, e \in \mathcal{U}_{m}^{0}\right\}
$$

The curl operator is well defined as $\mathcal{V}\left(\mathcal{T}^{\text {int }}, \mathcal{T}^{\ell}\right) \rightarrow \operatorname{ker}\left(\operatorname{div} ; W_{m, 0}^{2}\right)$. 
The curl mapping $\mathcal{V}\left(\mathcal{T}^{\text {int }}, \mathcal{T}^{\ell}\right) \rightarrow \operatorname{ker}\left(\operatorname{div} ; W_{m, 0}^{2}\right)$ is injective. The proof given for Proposition 6.1 is the same with $\mathbf{p}_{m}^{0} \in \mathcal{V}\left(\mathcal{T}^{\text {int }}, \mathcal{T}^{\ell}\right)$ of the form

$$
\mathbf{p}_{m}^{0}=\sum_{e \in \mathcal{U}_{m}^{0}} \hat{u}_{e} \mathbf{w}_{e}
$$

The linear spaces $\mathcal{V}\left(\mathcal{T}^{\text {int }}, \mathcal{T}^{\ell}\right)$ and $\operatorname{ker}\left(\operatorname{div} ; W_{m, 0}^{2}\right)$ have the same dimension. Because $\# \mathcal{T}^{\text {int }}=\left(N_{m}-N_{m}^{\Gamma}-1\right), \# \mathcal{T}^{\ell}=1$, on one hand we have

$$
\operatorname{dim}\left[\mathcal{V}\left(\mathcal{T}^{\text {int }}, \mathcal{T}^{\ell}\right)\right]=E_{m}-E_{m}^{\Gamma}-\left(N_{m}-N_{m}^{\Gamma}-1+1\right) .
$$

On the other hand, because $\operatorname{dim}\left[W_{m}^{2}\right]=F_{m}$ and $\operatorname{dim}\left[W_{m, 0}^{2}\right]=F_{m}-F_{m}^{\Gamma}$, we get

$$
\operatorname{dim}\left[\operatorname{ker}\left(\operatorname{div} ; W_{m, 0}^{2}\right)\right]=F_{m}-F_{m}^{\Gamma}-\left(T_{m}-1\right) .
$$

Note that we have $T_{m}-1$ independent relations since $\operatorname{div} \mathbf{v}=0$ and $\Omega$ is simply connected, as it is proved in Lemma 4.2 of [10]. By using the Euler-Poincaré characteristics and Remarks 4.1 and 4.2 , we get

$$
\begin{aligned}
E_{m} & -E_{m}^{\Gamma}-\left(N_{m}-N_{m}^{\Gamma}\right)-\left(F_{m}-F_{m}^{\Gamma}-\left(T_{m}-1\right)\right) \\
= & -\left(N_{m}-E_{m}+F_{m}-T_{m}\right)+\left(N_{m}^{\Gamma}-E_{m}^{\Gamma}+F_{m}^{\Gamma}\right)-1 \\
\quad & =-1+2-1=0 .
\end{aligned}
$$

The present proof can also be carried out at an algebraic level. Proposition 6.2 states that, given $\mathcal{T}^{\text {int }} \cup \mathcal{T}^{\ell}, \omega \in \operatorname{ker}\left(\operatorname{div} ; W_{m}^{2}\right)$, and $\hat{\mathbf{u}}_{m}$ the divergence-free lifting in $\Omega$ of $\hat{\mathbf{u}}_{m}^{\Gamma}$ defined in Proposition 6.1, there is a unique divergence-free vector $\mathbf{u}_{m}^{0} \in W_{m, 0}^{2}$ of the form (16). Moreover, its coefficients $u_{e}^{0}, e \in \mathcal{U}_{m}^{0}$, on the chosen basis, are the components of the solution of the linear system

$$
\sum_{e \in \mathcal{U}_{m}^{0}} u_{e}^{0} \int_{\Omega} \operatorname{curl}_{e} \cdot \operatorname{curl}_{\gamma}=\int_{\Omega} \omega \cdot \mathbf{w}_{\gamma}-\int_{\Omega} \hat{\mathbf{u}}_{m} \cdot \mathbf{w}_{\gamma} \quad \forall \gamma \in \mathcal{U}_{m}^{0} .
$$

The matrix

$$
A=\left(\int_{\Omega} \operatorname{curl} \mathbf{w}_{e} \cdot \operatorname{curl} \mathbf{w}_{\gamma}\right)_{e, \gamma \in \mathcal{U}_{m}^{0}}
$$

has full rank; it is in fact the mass matrix for the chosen basis $\left\{\right.$ curl $\left._{e} \mid e \in \mathcal{U}_{m}^{0}\right\}$ (defined on the coedges) of the space $\operatorname{ker}\left(\operatorname{div} ; W_{m, 0}^{2}\right)$. Note that $A$ is a symmetric and positive definite sparse matrix so that the linear system (18) can be solved iteratively by using a conjugate gradient method, as first done by Roux [28].

Remark 6.3. Note that $\mathcal{E}_{m}^{\text {int }}$ and $\mathcal{E}_{m}^{\ell}$ can be empty. In this case, there is no interior spanning tree $\left(\mathcal{T}^{\text {int }} \cup \mathcal{T}^{\ell}\right)$ and $\mathcal{U}_{m}^{0}=\mathcal{E}_{m}^{B}$. As $N_{m}=N_{m}^{\Gamma}$, Remarks 4.1 and 4.2 yield again

$$
\left(\# \mathcal{U}_{m}^{0}=\right) \quad E_{m}-E_{m}^{\Gamma}=F_{m}-F_{m}^{m}-\left(T_{m}-1\right) \quad\left(=\operatorname{dim}\left[\operatorname{ker}\left(\operatorname{div} ; W_{m, 0}^{2}\right)\right]\right) .
$$

A similar remark can be done in the nonsimply connected case.

The function

$$
\mathbf{u}_{m}=\hat{\mathbf{u}}_{m}+\sum_{e \in \mathcal{U}_{m}^{0}} u_{e}^{0} \operatorname{curl} \mathbf{w}_{e}
$$

is then the approximated solution of problem (7). It is natural to ask whether the computed solution depends on the chosen spanning tree. The answer is no, as we state in the next subsection. 
6.3. Independence on the spanning tree. We remark that the solution does not depend on the adopted spanning tree if $\omega \in \operatorname{ker}\left(\operatorname{div} ; W_{m}^{2}\right)$. Let us consider two boundary and interior spanning trees as well as two sets of mesh edges:

$$
\begin{array}{lll}
\mathcal{T}_{1}^{\Gamma}, & \mathcal{T}_{1}=\mathcal{T}_{1}^{\text {int }} \cup \mathcal{T}_{1}^{\ell}, & \mathcal{U}_{m, 1}^{0}=\left(\mathcal{E}_{m}^{\text {int }} \cup \mathcal{E}_{m}^{\ell} \cup \mathcal{E}_{m}^{B}\right) \backslash \mathcal{T}_{1}, \\
\mathcal{T}_{2}^{\Gamma}, & \mathcal{T}_{2}=\mathcal{T}_{2}^{\text {int }} \cup \mathcal{T}_{2}^{\ell}, & \mathcal{U}_{m, 2}^{0}=\left(\mathcal{E}_{m}^{\text {int }} \cup \mathcal{E}_{m}^{\ell} \cup \mathcal{E}_{m}^{B}\right) \backslash \mathcal{T}_{2} .
\end{array}
$$

Let $\mathbf{u}_{m}^{i}$ be the solution associated with $\mathcal{E}_{m}^{\Gamma} \backslash \mathcal{T}_{i}^{\Gamma}$ on the boundary and with $\mathcal{U}_{m, i}^{0}$ at the interior $(i=1,2)$. Let us denote $\mathbf{v}_{m}=\mathbf{u}_{m}^{1}-\mathbf{u}_{m}^{2}$. We will prove that $\mathbf{v}_{m}=\mathbf{0}$.

On the boundary we consider $\mathbf{v}_{m}=\sum_{e \in \mathcal{E}_{m}^{\Gamma}} v_{a} \operatorname{curl} \mathbf{w}_{e} ;$ since $\mathbf{u}_{m}^{1} \cdot \mathbf{n}_{\Gamma}=\mathbf{u}_{m}^{2} \cdot \mathbf{n}_{\Gamma}=g$ on $\Gamma$, we have

$$
\int_{f} \mathbf{v}_{m} \cdot \mathbf{n}_{\Gamma}=0 \quad \forall f \in \mathcal{F}_{m}^{\Gamma}
$$

Thus $\mathbf{v}_{m} \cdot \mathbf{n}_{\Gamma}=0$ on $\Gamma$, since the family $\left\{\operatorname{curl}_{e} \mid \mathbf{w}_{e} \in W_{m}^{1}, e \in \mathcal{E}_{m}^{\Gamma} \backslash \mathcal{T}_{i}^{\Gamma}\right\}, i=1$ or 2 , is a basis for the space $\operatorname{span}\left\{\operatorname{curl} \mathbf{w}_{e} \mid \mathbf{w}_{e} \in W_{m}^{1}, e \in \mathcal{E}_{m}^{\Gamma}\right\}$.

In the interior, because $\omega \in \operatorname{ker}\left(\operatorname{div} ; W_{m}^{2}\right)$, we can write that

$$
\omega=\sum_{e \in \mathcal{E}_{m}} \omega_{e} \operatorname{curl} \mathbf{w}_{e}, \quad \mathbf{w}_{e} \in W_{m}^{1}
$$

We have that, for all $\mathbf{w}_{\gamma} \in W_{m}^{1}$ with $\gamma \in \mathcal{U}_{m, 1}^{0}$ or $\gamma \in \mathcal{U}_{m, 2}^{0}$,

$$
\int_{\Omega} \omega \cdot \mathbf{w}_{\gamma}=\sum_{e \in \mathcal{E}_{m}} \omega_{e} \int_{\Omega} \mathbf{w}_{e} \cdot \operatorname{curl} \mathbf{w}_{\gamma} .
$$

The family $\left\{\operatorname{curl}_{\mathbf{w}} \mid \mathbf{w}_{\gamma} \in W_{m}^{1}, \gamma \in \mathcal{U}_{m, i}^{0}\right\}, i=1$ or 2 , is a basis for $\operatorname{ker}\left(\operatorname{div} ; W_{m}^{2}\right)$, so we have that for all $\gamma \in \mathcal{E}_{m}^{\text {int }} \cup \mathcal{E}_{m}^{\ell} \cup \mathcal{E}_{m}^{B}$ and not only for all $\gamma \in \mathcal{U}_{m, i}^{0}$,

$$
\int_{\Omega} \omega \cdot \mathbf{w}_{\gamma}=\sum_{e \in \mathcal{E}_{m}} \omega_{e} \int_{\Omega} \mathbf{w}_{e} \cdot \operatorname{curl} \mathbf{w}_{\gamma}
$$

Remarking that for all $\gamma \in \mathcal{E}_{m}^{\text {int }} \cup \mathcal{E}_{m}^{\ell} \cup \mathcal{E}_{m}^{B}$

$$
\begin{aligned}
& \int_{\Omega} \mathbf{u}_{m}^{1} \cdot \operatorname{curl} \mathbf{w}_{\gamma}=\int_{\Omega} \omega \cdot \mathbf{w}_{\gamma}, \\
& \int_{\Omega} \mathbf{u}_{m}^{2} \cdot \operatorname{curl} \mathbf{w}_{\gamma}=\int_{\Omega} \omega \cdot \mathbf{w}_{\gamma},
\end{aligned}
$$

we have that, for all $\gamma \in \mathcal{E}_{m}^{\text {int }} \cup \mathcal{E}_{m}^{\ell} \cup \mathcal{E}_{m}^{B}$,

$$
\int_{\Omega} \mathbf{v}_{m} \cdot \operatorname{curl} \mathbf{w}_{\gamma}=0
$$

That together with $\mathbf{v}_{m} \in \operatorname{ker}\left(\operatorname{div} ; W_{m}^{2}\right)$ implies $\mathbf{v}_{m}=\mathbf{0}$.

As we have seen, the final solution does not depend on the particular spanning tree to gauge the potential. In practice, however, the efficiency of the method does via the dependence on the particular tree of the condition number of the "stiffness" matrix $A$ in (18). This dependence is not too dramatic anyway, as underlined by the numerical tests presented in the appendix of [10]. 


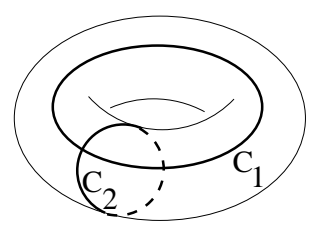

FIG. 5. Two disjoint loops $\mathcal{C}_{1}$ and $\mathcal{C}_{2}$, an example of generators of the first homology group $H_{1}\left(m^{\Gamma}\right)$ of the torus surface $\Gamma$.

7. Approximation of the problem in the nonsimply connected case. In the following, we turn our attention to the case where $\Omega$ and its boundary are nonsimply connected. As an example of such a situation, we assume that $\Omega$ is a torus, up to a homeomorphism. For the torus, we have $\chi(\Gamma)=0$ and $\chi(\Omega)=0$. Note that $\Gamma$ is an "empty" torus, while $\Omega$ is a "full" torus. Let us look at the differences between the simply connected and nonsimply connected cases.

7.1. Lifting the boundary condition for a torus. Let $\mathcal{S}^{\Gamma}$ denote the belted spanning tree on the torus boundary, i.e., $\mathcal{S}^{\Gamma}=\mathcal{T}^{\Gamma} \cup\left\{\Pi_{1}, \Pi_{2}\right\}$, where $\mathcal{T}^{\Gamma}$ is the usual spanning tree without loops and $\Pi_{1}, \Pi_{2}$ are two suitable edges of $\mathcal{E}_{m}^{\Gamma}$. In particular, denote $\mathcal{C}_{1}$ and $\mathcal{C}_{2}$ two disjoint loops of $\Gamma$, as presented in Figure 5 , and we have that

$$
\begin{aligned}
& \mathcal{T}^{\Gamma} \cup\left\{\Pi_{1}\right\} \text { contains a loop homologous to } \mathcal{C}_{1}, \\
& \mathcal{T}^{\Gamma} \cup\left\{\Pi_{2}\right\} \text { contains a loop homologous to } \mathcal{C}_{2} .
\end{aligned}
$$

Note that, with respect to the simply connected case, the spanning tree on the surface has been enriched according to $\operatorname{dim}\left[H_{1}\left(m^{\Gamma}\right)\right]$ (two edges in the case of the torus surface), as explained in Remark 5.1. Thanks to these added edges, the circuits associated to all remaining coedges do bound a surface contained in $\Gamma$, and this is a property that will be exploited during the proof of the following proposition. See [27] for a method to build up a belted spanning tree.

Proposition 7.1. Let us consider a triangulation $m$ of $\bar{\Omega}$, its restriction $m^{\Gamma}$ to the boundary $\Gamma$ of $\Omega$, and a spanning tree $\mathcal{S}^{\Gamma}$ in $m^{\Gamma}$. Let $\Gamma$ be a torus, and let $g \in L_{0}^{2}(\Gamma)$. There is a unique divergence-free vector $\hat{\mathbf{u}}_{m}^{\Gamma} \in W_{m^{\Gamma}}^{2}$ of the form

$$
\hat{\mathbf{u}}_{m}^{\Gamma}=\sum_{e \in \mathcal{E}_{m}^{\Gamma} \backslash \mathcal{S}^{\Gamma}} \hat{u}_{e} \operatorname{curl} \mathbf{w}_{e}, \quad \mathbf{w}_{e} \in W_{m^{\Gamma}}^{1},
$$

that satisfies

$$
\int_{f} \hat{\mathbf{u}}_{m}^{\Gamma} \cdot \mathbf{n}_{\Gamma}=\int_{f} g \quad \forall f \in \mathcal{F}_{m}^{\Gamma}
$$

Proof. The proof is similar to the proof of Proposition 6.1. We introduce the two spaces $\operatorname{ker}\left(\operatorname{div}_{\Gamma} ; W_{m^{\Gamma}}^{2}\right)$ and

$$
\mathcal{V}\left(\mathcal{S}^{\Gamma}\right)=\operatorname{span}\left\{\mathbf{w}_{e} \mid \mathbf{w}_{e} \in W_{m^{\Gamma}}^{1}, e \in \mathcal{E}_{m}^{\Gamma} \backslash \mathcal{S}^{\Gamma}\right\} .
$$

The curl operator is well defined as $\mathcal{V}\left(\mathcal{S}^{\Gamma}\right) \rightarrow \operatorname{ker}\left(\operatorname{div}_{\Gamma} ; W_{m^{\Gamma}}^{2}\right)$.

The curl mapping $\mathcal{V}\left(\mathcal{S}^{\Gamma}\right) \rightarrow \operatorname{ker}\left(\operatorname{div}_{\Gamma} ; W_{m^{\Gamma}}^{2}\right)$ is injective. Let us consider the vector $\hat{\mathbf{p}}_{m}^{\Gamma} \in \mathcal{V}\left(\mathcal{S}^{\Gamma}\right)$ of the form

$$
\hat{\mathbf{p}}_{m}^{\Gamma}=\sum_{e \in \mathcal{E}_{m}^{\Gamma} \backslash \mathcal{S}^{\Gamma}} \hat{u}_{e} \mathbf{w}_{e}
$$




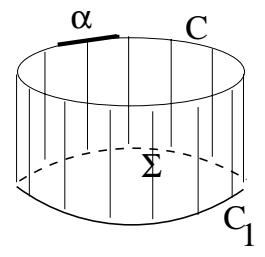

FIG. 6. When the circuit $\mathcal{C}$ is homologous to one of the two independent loops, say, $\mathcal{C}_{1}$, it does not bound any surface. In this case, we have to consider the loop $\mathcal{C} \cup \mathcal{C}_{1}$ that bounds a (lateral, in this case) surface $\Sigma$.

Let $\alpha \in \mathcal{E}_{m}^{\Gamma} \backslash \mathcal{S}^{\Gamma}$ be a given coedge, and let $\mathcal{C} \subset\{\alpha\} \cup \mathcal{S}^{\Gamma}$ be the associated cycle. It can happen either that $\mathcal{C}$ is the boundary of a surface $\Sigma$ (in this case we repeat exactly the proof for the simply connected case) or that $\mathcal{C}$ is homologous to one of the two fundamental loops, say, $\mathcal{C}_{1}$, and so it does not bound a surface. To overcome the problem, we have to consider the loop $\mathcal{C} \cup \mathcal{C}_{1}$; now, this loop does bound a surface, and we denote by $\Sigma$ the corresponding surface (see Figure 6 ).

We have then

$$
\int_{\mathcal{C} \cup \mathcal{C}_{1}} \hat{\mathbf{p}}_{m}^{\Gamma} \cdot \tau_{\mathcal{C} \cup \mathcal{C}_{1}}=\hat{u}_{\alpha} \int_{\alpha} \mathbf{w}_{\alpha} \cdot \tau_{\mathcal{C} \cup \mathcal{C}_{1}}=\hat{u}_{\alpha},
$$

where $\tau_{\mathcal{C} \cup \mathcal{C}_{1}}$ is the tangential vector to $\mathcal{C} \cup \mathcal{C}_{1}$. We then conclude that $\hat{u}_{\alpha}=0$ by following the same steps of the proof for Proposition 6.1.

Let $W$ again be the space composed of vectors in $\operatorname{ker}\left(\operatorname{div}_{\Gamma} ; W_{m^{\Gamma}}^{2}\right)$ verifying (21) with $g \in L_{0}^{2}(\Gamma)$. The linear spaces $\mathcal{V}\left(\mathcal{S}^{\Gamma}\right)$ and $W$ have the same dimension. On one hand, we have $\operatorname{dim}\left[\mathcal{V}\left(\mathcal{S}^{\Gamma}\right)\right]=E_{m}^{\Gamma}-\left(N_{m}^{\Gamma}-1+2\right)$ thanks to Remark 5.1, and on the other hand, $\operatorname{dim}[W]=F_{m}^{\Gamma}-1$. Due to the fact that the Euler-Poincaré characteristic for an empty torus is 0 , the equality

$$
E_{m}^{\Gamma}-N_{m}^{\Gamma}-1=F_{m}^{\Gamma}-1
$$

follows from Remark 4.2.

7.2. Interior problem for a torus. Let $\mathcal{C}_{1}$ be the loop that does not bound any surface of $\Omega$, and let $\mathcal{C}_{2}$ be the one that bounds a surface $\Sigma_{2}$ when considered in $\Omega$ (see Figure 5). The flux condition

$$
\int_{\Sigma_{2}} \mathbf{u} \cdot \mathbf{n}_{\Sigma}=\int_{\partial \Sigma_{2}} \mathbf{p} \cdot \tau_{\mathcal{C}_{2}} \neq 0
$$

yields $\pi \mathbf{p}$ not identically null. In this case, to solve problem (9) we need to "reactivate" one of the two edges $\Pi_{1}, \Pi_{2}$ excluded in problem (8) and precisely the one associated with the loop that bounds when we pass from $\Gamma$ to $\Omega$. In any other case, the degree of freedom associated to $\Pi_{2}$ is zero. In the following, we take into account the more general case where $\pi \mathbf{p} \neq \mathbf{0}$ and we assume that $\Pi_{2}^{*}=\operatorname{supp}(\pi \mathbf{p})$. The degree of freedom associated to this particular edge is equal to the flux of the field $\mathbf{u}$ across the transversal section of the torus. For this feature, from now on, we call $\Pi_{2}^{*}$ the "flux edge." Denoting by $\mathcal{T}^{\text {int }}$ the usual spanning tree without loops, we have $\mathcal{S}^{\text {int }}=$ $\mathcal{T}^{\text {int }} \cup\left\{\Pi_{1}\right\}$, and the set $\mathcal{U}_{m}^{0}$ is now defined as follows:

$$
\tilde{\mathcal{U}}_{m}^{0}=\left(\mathcal{E}_{m}^{\text {int }} \cup \mathcal{E}_{m}^{\ell} \cup \mathcal{E}_{m}^{B} \cup\left\{\Pi_{2}^{*}\right\}\right) \backslash\left(\mathcal{S}^{\text {int }} \cup \mathcal{T}^{\ell}\right) .
$$


Proposition 7.2. Let us consider a triangulation $m$ of $\bar{\Omega}$, together with a spanning tree $\mathcal{S}^{\text {int }} \cup \mathcal{T}^{\ell}$ in $m$. Let us suppose that $\Omega$ is a torus and $\omega \in \operatorname{ker}\left(\operatorname{div} ; W_{m}^{2}\right)$. There exists a unique divergence-free vector $\mathbf{u}_{m}^{0} \in W_{m, 0}^{2}$ of the form

$$
\mathbf{u}_{m}^{0}=\sum_{e \in \tilde{\mathcal{U}}_{m}^{0}} u_{e}^{0} \operatorname{curl} \mathbf{w}_{e} \quad \mathbf{w}_{e} \in W_{m}^{1},
$$

that satisfies

$$
\operatorname{curl} \mathbf{u}_{m}^{0}=\omega-\operatorname{curl} \hat{\mathbf{u}}_{m} \quad \text { in } \Omega,
$$

where $\hat{\mathbf{u}}_{m}$ is the divergence-free lifting in $\Omega$ of $\hat{\mathbf{u}}_{m}^{\Gamma}$ defined in Proposition 7.1.

Proof. Let us introduce the two spaces $\operatorname{ker}\left(\operatorname{div} ; W_{m, 0}^{2}\right)$ and

$$
\mathcal{V}\left(\mathcal{S}^{\text {int }}, \mathcal{T}^{\ell}\right)=\operatorname{span}\left\{\mathbf{w}_{e} \mid \mathbf{w}_{e} \in W_{m}^{1}, e \in \tilde{\mathcal{U}}_{m}^{0}\right\}
$$

The curl operator is well defined as $\mathcal{V}\left(\mathcal{S}^{\text {int }}, \mathcal{T}^{\ell}\right) \rightarrow \operatorname{ker}\left(\operatorname{div} ; W_{m, 0}^{2}\right)$.

The curl mapping $\mathcal{V}\left(\mathcal{S}^{\text {int }}, \mathcal{T}^{\ell}\right) \rightarrow \operatorname{ker}\left(\operatorname{div} ; W_{m, 0}^{2}\right)$ is injective. The proof given for Proposition 7.1 is the same with $\mathbf{p}_{m}^{0} \in \mathcal{V}\left(\mathcal{S}^{\text {int }}, \mathcal{T}^{\ell}\right)$ of the form

$$
\mathbf{p}_{m}^{0}=\sum_{e \in \tilde{\mathcal{U}}_{m}^{0}} \hat{u}_{e} \mathbf{w}_{e}
$$

The linear spaces $\mathcal{V}\left(\mathcal{S}^{\operatorname{int}}, \mathcal{T}^{\ell}\right)$ and $\operatorname{ker}\left(\operatorname{div} ; W_{m, 0}^{2}\right)$ have the same dimension. In fact, we have

$$
\begin{aligned}
& \operatorname{dim}\left[\mathcal{V}\left(\mathcal{S}^{\text {int }}, \mathcal{T}^{\ell}\right)\right]=E_{m}-E_{m}^{\Gamma}+1-\left(N_{m}-N_{m}^{\Gamma}-1+1+1\right), \\
& \operatorname{dim}\left[\operatorname{ker}\left(\operatorname{div} ; W_{m, 0}^{2}\right)\right]=F_{m}-F_{m}^{\Gamma}-\left(T_{m}-1\right)-1 .
\end{aligned}
$$

Note that now, for the presence of "one hole" in $\Omega$, the equation $\operatorname{div} \mathbf{u}=0$ gives only $T_{m}$ independent conditions. The two are coincident since the Euler-Poincaré characteristic for the "full" torus and its surface is 0 .

Once again, the present proof can also be carried out at an algebraic level. Proposition 7.2 states that, given $\mathcal{S}^{\text {int }} \cup \mathcal{T}^{\ell}, \omega \in \operatorname{ker}\left(\operatorname{div} ; W_{m}^{2}\right)$, and $\hat{\mathbf{u}}_{m}$ the divergence-free lifting in $\Omega$ of $\hat{\mathbf{u}}_{m}^{\Gamma}$ defined in Proposition 7.1, there is a unique divergence-free vector $\mathbf{u}_{m}^{0} \in W_{m, 0}^{2}$ of the form (22). Moreover, its coefficients $u_{e}^{0}, e \in \tilde{\mathcal{U}}_{m}^{0}$, on the chosen basis, are the components of the solution of the linear system

$$
\sum_{e \in \tilde{\mathcal{U}}_{m}^{0}} u_{e}^{0} \int_{\Omega} \operatorname{curl}_{e} \cdot \mathbf{c u r l}_{\gamma}=\int_{\Omega} \omega \cdot \mathbf{w}_{\gamma}-\int_{\Omega} \hat{\mathbf{u}}_{m} \cdot \mathbf{w}_{\gamma} \quad \forall \gamma \in \tilde{\mathcal{U}}_{m}^{0} .
$$

The matrix

$$
\tilde{A}=\left(\int_{\Omega} \operatorname{curl}_{e} \cdot \operatorname{curl}_{\gamma}\right)_{e, \gamma \in \tilde{\mathcal{U}}_{m}^{0}}
$$

again has full rank, but it is no more sparse due to the presence of the basis function associated to $\left\{\Pi_{2}^{*}\right\}$.

The function

$$
\mathbf{u}_{m}=\hat{\mathbf{u}}_{m}+\sum_{e \in \tilde{\mathcal{U}}_{m}^{0}} u_{e}^{0} \operatorname{curl} \mathbf{w}_{e}
$$

is then the approximated solution of problem (7) in the nonsimply connected case. 


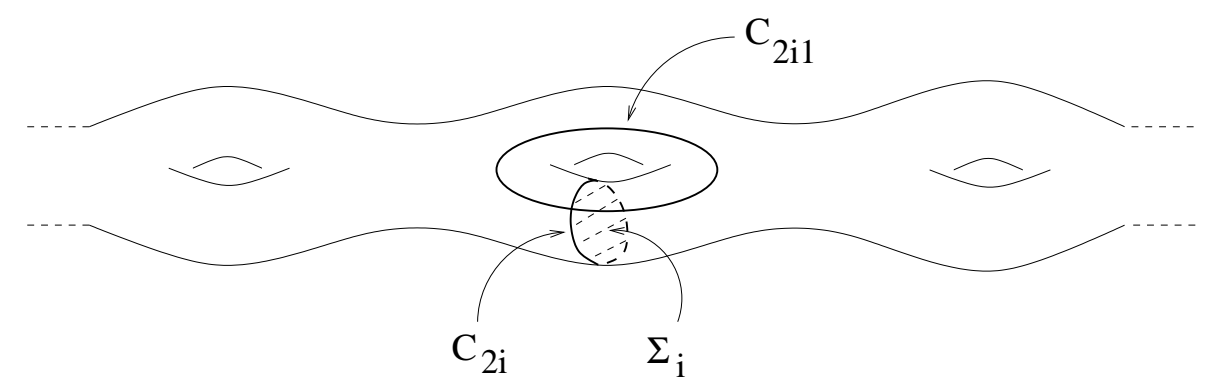

FIG. 7. Two disjoint loops $\mathcal{C}_{2 i-1}$ and $\mathcal{C}_{2 i}, 1 \leq i \leq \kappa$, example of generators of the first homology group of the ith "empty" torus belonging to the surface of the sum of $\kappa$ tori.

7.3. Case of the sum of $\kappa$ tori. The theory that we have presented can be generalized to a domain $\Omega$ that is the sum of $\kappa$ tori, with the integer $\kappa \geq 1$. We have that

$$
\chi(\Gamma)=1-2 \kappa+1=2(1-\kappa), \quad \chi(\Omega)=1-\kappa .
$$

For problem (8), we have to consider

$$
\mathcal{S}^{\Gamma}=\left(\mathcal{T}^{\Gamma} \cup \bigcup_{i=1}^{\kappa}\left\{\Pi_{2 i-1}, \Pi_{2 i}\right\}\right),
$$

where $\mathcal{T}^{\Gamma}$ is the usual spanning tree without loops and $\left\{\Pi_{2 i-1}, \Pi_{2 i}\right\}$ for $1 \leq i \leq \kappa$ is one pair of suitable edges of $\mathcal{E}_{m}^{\Gamma}$. In particular, denoting by $\mathcal{C}_{2 i-1}$ and $\mathcal{C}_{2 i}$ two disjoint loops as presented in Figure 7, we have that

$$
\begin{aligned}
& \mathcal{T}^{\Gamma} \cup\left\{\Pi_{2 i-1}\right\} \text { contains a loop homologous to } \mathcal{C}_{2 i-1}, \\
& \mathcal{T}^{\Gamma} \cup\left\{\Pi_{2 i}\right\} \text { contains a loop homologous to } \mathcal{C}_{2 i} .
\end{aligned}
$$

The spanning tree on the surface has been enriched according to $\operatorname{dim}\left[H_{1}\left(m^{\Gamma}\right)\right]$ that is now $2 \kappa$, as explained in Remark 5.1. The proof of Proposition 7.1 for problem (8) does not change globally; concerning the dimension of the approximation and approximated spaces, we have now

$$
\operatorname{dim}\left[\mathcal{V}\left(\mathcal{S}^{\Gamma}\right)\right]=E_{m}^{\Gamma}-\left(N_{m}^{\Gamma}-1+2 \kappa\right), \quad \operatorname{dim}[W]=F_{m}^{\Gamma}-1
$$

The two coincide thanks to the Euler-Poincaré characteristic of $\Gamma$.

Similarly, for $1 \leq i \leq \kappa$, let $\mathcal{C}_{2 i-1}$ be the loop that does not bound any surface of $\Omega$, and let $\mathcal{C}_{2 i}$ be the one that bounds a surface $\Sigma_{i}$ when considered in $\Omega$ (see Figure 7). For problem (9) we need to "reactivate" one of the two edges $\Pi_{2 i-1}, \Pi_{2 i}$ excluded in problem (8) and precisely the one associated with the loop that bounds a surface when we pass from $\Gamma$ to $\Omega$. So, because $\Pi_{2 i}^{*}=\operatorname{supp}(\pi \mathbf{p})$, the flux edges, $1 \leq i \leq \kappa$, with the vector $\mathbf{p}$ as in section 7.2 , and denoting by $\mathcal{T}^{\text {int }}$ the usual spanning tree without loops, we have

$$
\mathcal{S}^{\text {int }}=\left(\mathcal{T}^{\text {int }} \cup \bigcup_{i=1}^{\kappa}\left\{\Pi_{2 i-1}\right\}\right),
$$


and the set $\mathcal{U}_{m}^{0}$ is now defined as follows:

$$
\tilde{\mathcal{U}}_{m}^{0}=\left(\mathcal{E}_{m}^{\text {int }} \cup \mathcal{E}_{m}^{\ell} \cup \mathcal{E}_{m}^{B} \cup \bigcup_{i=1}^{\kappa}\left\{\Pi_{2 i}^{*}\right\}\right) \backslash\left(\mathcal{S}^{\text {int }} \cup \mathcal{T}^{\ell}\right) .
$$

The proof of Proposition 7.2 for problem (9) is unchanged; the dimension of the approximation and approximated spaces is now

$$
\begin{aligned}
& \operatorname{dim}\left[\mathcal{V}\left(\mathcal{S}^{\mathrm{int}}, \mathcal{T}^{\ell}\right)\right]=E_{m}-E_{m}^{\Gamma}+\kappa-\left(N_{m}-N_{m}^{\Gamma}-1+\kappa+1\right), \\
& \operatorname{dim}\left[H_{0}\left(\operatorname{div}_{0}, m\right)\right]=F_{m}-F_{m}^{\Gamma}-\left(T_{m}-1\right)-\kappa,
\end{aligned}
$$

and the two coincide thanks to the Euler-Poincaré characteristic of $\Omega$ and $\Gamma$.

Remark 7.3. Another existing strategy to deal with potential problems in nonsimply connected domains relies on the introduction of "cuts" in the domain. The big difficulty with this method is the construction of cuts and understanding where they should be introduced. Kotiuga [21] and coworkers have provided a correct definition of a cut, a constructive algorithm, and an implementation of it (see [18], for example).

The "belted tree" approach proposed in this paper allows us to achieve knowledge of the topological features of the considered domain if this is not given a priori. This knowledge is a preliminary step to the introduction of cuts.

8. Algorithmics and a simple example. From a practical point of view, the determination of the set $\mathcal{U}_{m}^{0}$ for simply connected domains is standard, and we refer to [28] for a procedure to construct a particular spanning tree.

This is not the case for the nonsimply connected case. The problem is now to find out the independent loops in order to select explicitly the flux edges previously introduced. We have, in particular, to select the loops that bound a surface when we pass from the boundary to the interior. This question can be summarized by saying that we look for generators of $H_{1}(m)$ starting from those of $H_{1}\left(m^{\Gamma}\right)$.

In section 8.1, we present the algebraic tools, and we explain how to use them in section 8.2. The very first results for a torus are presented in section 8.3. We remark that only the computation of a basis for $H_{1}(m)$ is useful to our purpose of solving problem (24). In any case, a basis for $H_{2}(m)$ can be computed with the same tools, and at the end of section 8.2 we give a few indications of how to proceed with it. See [20] for another type of algorithm.

8.1. An integer $\mathcal{Q R}$ factorization. In this section, we present a matrix decomposition to compute a set of generators of the homology groups of order $p=1$ and 2 of $\Omega \subset \mathbb{R}^{3}$. The same algorithm has been used in [25] to detect mesh defects. The basic idea is to make an integer $\mathcal{Q R}$ factorization of the matrices $G^{t}, R^{t}$, and $D^{t}$. Given a matrix $A \in \mathcal{M}(r, s)$, we compute a nonsingular unimodular matrix $\mathcal{Q}$ (i.e., $\operatorname{det}(\mathcal{Q})= \pm 1$ ) and a permutation matrix $\mathcal{P}$ such that $\mathcal{R}=\mathcal{Q} A \mathcal{P}$ is upper triangular. As shown later, the two matrices $\mathcal{Q}$ and $\mathcal{P}$ are obtained as products of a certain number of local matrices $\mathcal{Q}_{i, j}$ and $\mathcal{P}_{i, j}$ and exhibit the row and column rank deficiency of $A$ [8]. The key point of the algorithm is the following property [17]: given a matrix $A \in \mathcal{M}(r, s)$ with integer elements, we have

$$
Z^{r}=\operatorname{ker}\left(A^{t}\right) \oplus \operatorname{range}(A), \quad Z^{s}=\operatorname{ker}(A) \oplus \operatorname{range}\left(A^{t}\right) .
$$

To define $\mathcal{Q}$ and $\mathcal{P}$, we need two elementary operations. First is the transformation $\pi_{1}$ of a vector $v=\left(\epsilon_{i}, \epsilon_{j}\right)^{t}$ into the vector $\tilde{v}=(1,0)^{t}$. To this purpose, let us introduce 
the elementary matrices

$$
\mathcal{Q}_{i, j}^{\mathrm{el}}=\left(\begin{array}{cc}
\epsilon_{i} & 0 \\
-\epsilon_{i} & \epsilon_{j}
\end{array}\right), \quad\left(\mathcal{Q}_{i, j}^{\mathrm{el}}\right)^{-1}=\left(\begin{array}{cc}
\epsilon_{i} & 0 \\
\epsilon_{j} & \epsilon_{j}
\end{array}\right)
$$

and the matrix

$$
\mathcal{Q}_{i, j}(\ell, q)=\left\{\begin{array}{lll}
\delta_{\ell, q}, & \ell \neq i, j, & q \neq i, j, \\
\mathcal{Q}_{i, j}^{\mathrm{el}}(1,1), & \ell=i, & q=i, \\
\mathcal{Q}_{i, j}^{\mathrm{el}}(1,2), & \ell=i, & q=j, \\
\mathcal{Q}_{i, j}^{\mathrm{el}}(2,1), & \ell=j, & q=i, \\
\mathcal{Q}_{i, j}^{\mathrm{el}}(2,2), & \ell=j, & q=j .
\end{array}\right.
$$

In our case, $\epsilon_{i}^{2}=1$, and the vector $\tilde{v}=\pi_{1}(v)=\mathcal{Q}_{i, j}^{\text {el }} v$. Second, we need the permutation $\pi_{2}$ of a vector's components, i.e., the transformation of a vector $v=\left(\epsilon_{i}, \epsilon_{j}\right)^{t}$ into the vector $\tilde{v}=\left(\epsilon_{j}, \epsilon_{i}\right)^{t}$. To this purpose, we have $\tilde{v}=\pi_{2}(v)=\mathcal{P}_{i, j}^{\mathrm{el}} v$, where $\mathcal{P}_{i, j}^{\mathrm{el}}$ is a permutation matrix; moreover, we introduce a matrix $\mathcal{P}_{i, j}$ defined similarly to $\mathcal{Q}_{i, j}$ (using $\mathcal{P}_{i, j}^{\mathrm{el}}$ instead of $\mathcal{Q}_{i, j}^{\mathrm{el}}$ ). We remark that $\left(\mathcal{P}_{i, j}^{\mathrm{el}}\right)^{-1}=\mathcal{P}_{i, j}^{\mathrm{el}}$, owing to the fact that $\mathcal{P}_{i, j}^{\text {el }}$ is a permutation matrix and that $\left(\mathcal{Q}_{i, j}\right)^{-1}$ is defined as $\mathcal{Q}_{i, j}\left(\operatorname{using}\left(\mathcal{Q}_{i, j}^{\mathrm{el}}\right)^{-1}\right.$ instead of $\mathcal{Q}_{i, j}^{\text {el }}$ ). In the following, $I_{r}$ denotes the identity matrix of dimension $r>0$. Now we describe the adopted procedure to build up $\mathcal{Q}$ and $\mathcal{P}$ for a given matrix $A \in \mathcal{M}(r, s)$.

Procedure. We set $\mathcal{Q}=\mathcal{Q}^{0} \in \mathcal{M}(r, r), \mathcal{P}=\mathcal{P}^{0} \in \mathcal{M}(s, s)$. We loop on the column index $j, 1 \leq j \leq s$ :

1. We define $\mathcal{V}_{j}=\{i \mid \min \{j, r\} \leq i \leq \min \{s, r\}, A(i, j) \neq 0\}$, and we put $k$ equal to the cardinality of $\mathcal{V}_{j}, i_{1}$ equal to the smallest integer in $\mathcal{V}_{j}$, and $i_{2}$ equal to the smallest integer in $\mathcal{V}_{j} \backslash\left\{i_{1}\right\}$.

2. In case $k=0$, let $\mathcal{P}_{j, z}$ be the matrix of the transformation $\pi_{2}$ that permutes the $j$ th column of $A$ with the $z$ th one. The $z$ th column is chosen to be the first column, starting from the last one in $A$, for which there exists a row index $s$ such that $A(s, z) \neq 0$. If the index $z$ exists, $\mathcal{P} \longleftarrow \mathcal{P} \mathcal{P}_{j, z}, A \longleftarrow A \mathcal{P}_{j, z}$, and we go back to step 1; otherwise we stop the procedure.

3 . In case $k \neq 0$ but $A(j, j)=0$, we apply a partial pivot strategy. Let $\mathcal{Q}_{j, i_{1}}$ be the matrix of the transformation $\pi_{2}$ that permutes the $j$ th row with the $i_{1}$ th one; then $\mathcal{Q} \longleftarrow \mathcal{Q}_{j, i_{1}} \mathcal{Q}, A \longleftarrow \mathcal{Q}_{j, i_{1}} A, i_{1} \longleftarrow j$, and we go to step 4 .

4. In case $k \geq 2$ and $A(j, j) \neq 0$, let $\mathcal{Q}_{i_{1}, i_{2}}^{\text {el }}$ be the matrix of the transformation $\pi_{1}$ applied to the vector $\left(A\left(i_{1}, j\right), A\left(i_{2}, j\right)\right)^{t}$, and let $\mathcal{Q}_{i_{1}, i_{2}}$ be the associated matrix; then $\mathcal{Q} \longleftarrow \mathcal{Q}_{i_{1}, i_{2}} \mathcal{Q}, A \longleftarrow \mathcal{Q}_{i_{1}, i_{2}} A$, and we go back to step 1 .

5. In case $k=1$ and $A(j, j) \neq 0$, then $j \longleftarrow j+1$, and we go back to step 1 .

Starting with $\mathcal{Q}^{0}=I_{r}$ and $\mathcal{P}^{0}=I_{s}$, at the end of the procedure, the matrix $A$ has been replaced by $\mathcal{R}$, an upper triangular one. If this new matrix $\mathcal{R}$ does not contain zero rows, then $\operatorname{dim}[\operatorname{range}(\mathcal{R})]=r$. Otherwise, $\operatorname{dim}\left[\operatorname{ker}\left(\mathcal{R}^{t}\right)\right]=r-\operatorname{dim}[\operatorname{range}(\mathcal{R})]$. We remark that the procedure converges and its computational cost is similar to that of a $\mathcal{Q R}$ decomposition by using Givens transformations.

8.2. Computation of homology group generators. Now, the question is how we can use the previous procedure to compute the generators of $H_{p}(m)$ for $p=1$ and 2. To compute a set of generators for $H_{1}(m)$, we proceed as follows.

(i) We apply the procedure with $A=R^{t}, \mathcal{Q}^{0}=I_{E_{m}}$, and $\mathcal{P}^{0}=I_{F_{m}}$, and we get two invertible matrices $\mathcal{Q}_{R}$ and $\mathcal{P}_{R}$ such that $\mathcal{R}_{R}=\mathcal{Q}_{R} R^{t} \mathcal{P}_{R}$ is upper 


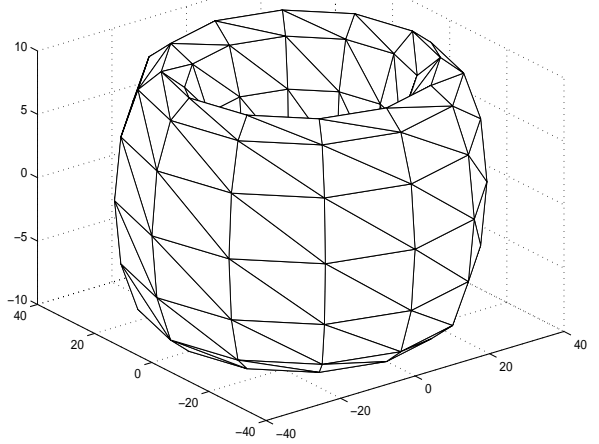

FIG. 8. An example of surface discretization for the torus.

triangular. The 1-cycles which bound a surface belong to the image of the matrix $R^{t}$ that is also the image of $\mathcal{R}_{R}$.

(ii) We define $\tilde{G}^{t}=G^{t} \mathcal{Q}_{R}^{-1}$. In this way we make a change of basis for the 1-chains. Looking at $\tilde{G}^{t}$, we see immediately from the presence of $n_{c}$ zero columns that the corresponding columns of $\mathcal{Q}_{R}^{-1}$ represent vectors that belong to the kernel of $G^{t}$. If $\operatorname{dim}\left[\operatorname{range}\left(R^{t}\right)\right]=\operatorname{dim}\left[\operatorname{ker}\left(G^{t}\right)\right]$, then any 1-cycle bounds. In the other case, we apply the procedure with $A=\tilde{G}^{t}, \mathcal{Q}^{0}=I_{N_{m}}$, and $\mathcal{P}^{0}=I_{E_{m}-n_{c}}$. We then obtain two invertible matrices $\mathcal{Q}_{\tilde{G}}$ and $\mathcal{P}_{\tilde{G}}$ such that $\mathcal{R}_{\tilde{G}}=\mathcal{Q}_{\tilde{G}} \tilde{G}^{t} \mathcal{P}_{\tilde{G}}$ is upper triangular.

(iii) The rows in $\mathcal{P}_{\tilde{G}}$, corresponding to zero rows in $\mathcal{R}_{\tilde{G}}$, represent the vectors that complete the kernel of $G^{t}$. In fact, we are looking for $c$ such that $G^{t} c=0$. This is equivalent to $\tilde{G}^{t} v=0$, where $v$ has zero in the first $n_{c}$ components and, in the last $E_{m}-n_{c}$, any row in $\mathcal{P}_{\tilde{G}}$ corresponding to a new zero row in $\mathcal{R}_{\tilde{G}}$. Then the components of $c=\mathcal{Q}_{R}^{-1} v$ are the coefficients of a 1-chain generator of $H_{1}(m)$.

To determine the generators of $H_{2}(m)$, it is sufficient to perform parts (i), (ii), and (iii) with $D^{t}$ at the place of $R^{t}$ and $R^{t}$ at the place of $G^{t}$.

8.3. Numerical results on the torus. As an application, we consider the case of a torus. We discretize it by means of a mesh $m$ of 596 tetrahedra and 179 nodes. The discretization of $\Omega$ induces a discretization of the surface, denoted $m^{\Gamma}$, composed of 288 triangles and 144 nodes (see Figure 8).

We apply the procedure presented in section 8.2 to the matrices $R^{t}$ and $G^{t}$ of the surface $\Gamma$. At this point we have a basis for the 1-cycles of the mesh $m^{\Gamma}$ that are not boundaries, i.e., a basis for $H_{1}\left(m^{\Gamma}\right)$ (see Figures 9 and 10). Note that the loops $\mathcal{C}_{1}$ and $\mathcal{C}_{2}$ run around the two "holes" of the torus surface.

We want now to make evident the loop that bounds a surface when considered as 1 -cycles of the mesh $m$ in $\Omega$. In other terms, in the set of the two computed generators for $H_{1}\left(m^{\Gamma}\right)$, we look for the one that generates $H_{1}(m)$.

Let $c$ be an element of $H_{1}\left(m^{\Gamma}\right)$ and $v_{c}$ the vector whose components are the coefficients of $c$ for the edges $e \in \mathcal{E}_{m}^{\Gamma}$ and zero for $e \in \mathcal{E}_{m} \backslash \mathcal{E}_{m}^{\Gamma}$. We apply the procedure to the matrix $R^{t}$ associated to the mesh $m$ in $\Omega$, and we transform it into an upper triangular matrix of the form $\mathcal{R}_{R}=\mathcal{Q}_{R} R^{t} \mathcal{P}_{R}$. Finally, we consider the vector $w_{c}=\mathcal{Q}_{R}^{-1} v_{c}$. If $w_{c}=0$, then the 1 -chain $c$ is homologous to zero in $\Omega$ (see 

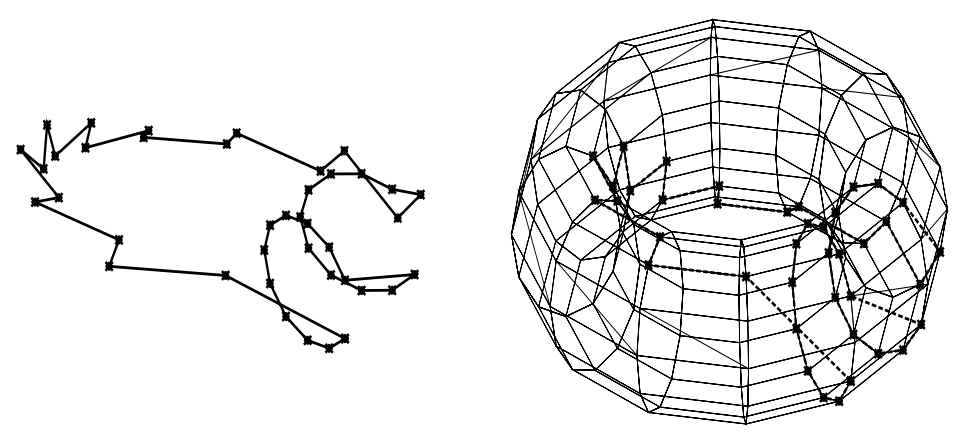

FIG. 9. Wireframe representation of the loop $\mathcal{C}_{2}$, one of the two generators of the first homology group of the torus surface.
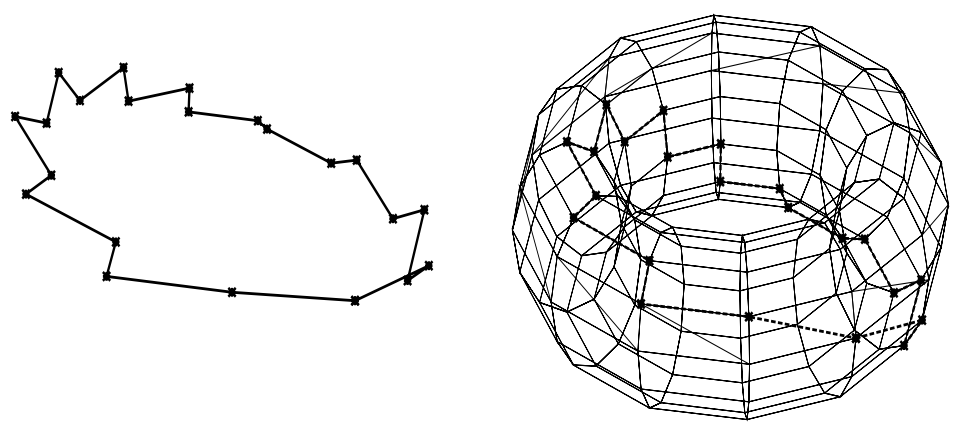

FIG. 10. Wireframe representation of the loop $\mathcal{C}_{1}$, one of the two generators of the first homology group of the torus surface.

Figure 9); if $w_{c} \neq 0$, then the 1-chain $c$ is also a generator of $H_{1}(m)$ (see Figure 10). In our case, the loop $\mathcal{C}_{1}$ is detected to be the element of a basis for $H_{1}(m)$. Note that the adopted procedure can be optimized in several ways, such as, for example, by looking for those generators with the minimum number of edges or faces, by applying the procedure to suitable portions of the whole meshes, and by using a suitable data format [13].

The two loops $\mathcal{C}_{1}$ and $\mathcal{C}_{2}$ are used as follows. For the lifting of the boundary condition, the set $\mathcal{S}^{\Gamma}$ of "null degrees of freedom" is a belted spanning tree obtained by adding to the standard spanning tree $\mathcal{T}^{\Gamma}$ two edges, $\Pi_{1} \in \mathcal{C}_{1}$ and $\Pi_{2} \in \mathcal{C}_{2}$, chosen in an arbitrary way, with $\Pi_{1} \neq \Pi_{2}$. These two edges correspond to nonzero components in the vector $w_{c}$ of the coefficients of the 1-chains $c$ that generate $H_{1}\left(m^{\Gamma}\right)$.

For the interior problem, the set of active edges $\tilde{\mathcal{U}}_{m}^{0}$ is obtained by adding a flux edge $\Pi_{2}^{*}$ to the set $\mathcal{U}_{m}^{0}$, the latter selected with the algorithm devoted to a simply connected domain. We conclude by remarking that $\Pi_{2}^{*}$, the edge that has to be reactivated when passing from the boundary to the interior to solve problem (24), can be chosen to be any edge $e \in \mathcal{E}_{m}^{\Gamma}$ for which the corresponding coefficient in the 1-chain $c$ is nonzero. 
9. Conclusions. In this paper, we have studied the representation of a solenoidal vector field in terms of a vector potential. The considered problem has been split into two parts - a lifting problem of the boundary condition and an internal problem with homogeneous boundary conditions.

The edge elements are a natural tool to compute vector potentials. On the other hand, the gauge condition, which is necessary to ensure the potential uniqueness, is taken into account in a fully discrete way and expressed in terms of a suitable set of active mesh edges (active in the sense that the associated degree of freedom is a priori different from zero).

According to the authors' knowledge, the problem of the computation of the vector potential is well understood for three-dimensional bounded domains which are connected and simply connected, even with a connected but nonsimply connected boundary. Here, we have presented a method to compute the vector potential for three-dimensional bounded domains which are connected but nonsimply connected, with a connected boundary. The case of three-dimensional bounded domains which are nonsimply connected with a nonconnected boundary has not been considered in the present work.

\section{REFERENCES}

[1] R. A. Adams, Sobolev Spaces, Academic Press, New York, 1975.

[2] C. Amrouche, C. Bernardi, M. Dauge, and V. Girault, Vector potentials in threedimensional nonsmooth domains, Math. Methods Appl. Sci., 21 (1998), pp. 823-864.

[3] A. Bendali, J. M. Dominguez, And S. Gallic, A variational approach for the vector potential formulation of the Stokes and Navier-Stokes problems in three dimensional domains, J. Math. Anal. Appl., 107 (1985), pp. 537-560.

[4] C. Bernardi, Méthodes d'éléments finis mixtes pour les équations de Navier-Stockes, Thèse du 3ème cycle, University of Paris, Paris, 1979.

[5] C. Bernardi, M. Dauge, and Y. Maday, Compatibilité de traces aux arêtes et coins d'un polyèdre, C. R. Acad. Sci. Paris Sér. I Math., 331 (2000), pp. 679-684.

[6] A. Bossavit, Computational Electromagnetism: Variational Formulations, Complementarity, Edge Elements, Academic Press, New York, 1998.

[7] A. Buffa ANd P. Ciarlet JR., On traces for functional spaces related to Maxwell's equation II: Hodge decompositions on the boundary of Lipschitz polyhedra and applications, Math. Methods Appl. Sci., 24 (2001), pp. 31-48.

[8] T. F. Chan, Rank revealing QR factorizations, Linear Algebra Appl., 88/89 (1987), pp. 67-82.

[9] Y. Choquet-Bruhat, Géometrie Différentielle et Systèmes Extérieurs, Dunod, Paris, 1968.

[10] F. DuBois, Discrete vector potential representation of a divergence-free vector field in threedimensional domains: Numerical analysis of a model problem, SIAM J. Numer. Anal., 27 (1990), pp. 1103-1141.

[11] F. Dubois and F. Rapetti, Du tourbillon au champ de vitesse, in Proceedings of the Workshop at the Conservatoire des Arts et Métiers de Paris on Modèles fluides et représentation en toubillons, Vol. 1, Paris, France, 2000, pp. 127-153; MATAPLI, 65 (2001), pp. 87-88.

[12] C. Foias and R. Temam, Remarques sur les équations de Navier-Stokes stationnaires et les phénomènes successifs de bifurcation, Ann. Scuola Norm. Sup. Pisa Cl. Sci., 5 (1978), pp. 29-63.

[13] L. Formaggia, personal communication, Ecole Polytechnique Féderale de Lausanne, Lausanne, France, 2002.

[14] P. J. Giblin, Graphs, Surfaces and Homology. An Introduction to Algebraic Topology, Chapman and Hall Mathematics Series, Chapman and Hall, London, 1977.

[15] V. Girault and P. A. Raviart, Finite element methods for Navier-Stokes equations: Theory and Applications, Springer-Verlag, New York, 1986.

[16] J. GLimm, personal communication, Department of Applied Mathematics and Statistics, University of Stony Brook, Stony Brook, NY, 1986.

[17] G. H. Golub and C. F. Van Loan, Matrix Computation, John Hopkins University Press, Baltimore, MD, 1983. 
[18] P. Gross And P. R. Kotiuga, Finite element-based algorithms to make cuts for magnetic scalar potentials: Topological constraints and computational complexity, in Geometric Methods for Computational Electromagnetics, Progress in Electromagnetic Research Ser. 32, F. L. Teixeira, ed., EMW, Cambridge, MA, 2001, pp. 207-245.

[19] F. Hеснт, Construction d'une base de fonctions $\mathbb{P}_{1}$ non conforme à divergence nulle dans $\mathbb{R}^{3}$, RAIRO Anal. Numer., 15 (1980), pp. 315-341.

[20] R. Hiptmair and J. Ostrowski, Generators of $H_{1}\left(\Gamma_{h}, Z\right)$ for Triangulated Surfaces: Construction and Classification, Sonderforschungsbereich 382, Report 160, Universität Tübingen, Tübingen, Germany, 2001.

[21] P. R. KotiugA, An algorithm to make cuts for magnetic scalar potentials in tetrahedral meshes based on the finite element method, IEEE Trans. Magnetics, 25 (1989), pp. 4129-4131.

[22] C. Mattiussi, An analysis of finite volume, finite element, and finite difference methods using some concepts from algebraic topology, J. Comput. Phys., 133 (1997), pp. 289-309.

[23] J. C. NÉdÉLEC, Mixed finite elements in $\mathbb{R}^{3}$, Numer. Math., 35 (1980), pp. 315-341.

[24] J. C. NÉdÉlec, A new family of mixed finite elements in $\mathbb{R}^{3}$, Numer. Math., 35 (1986), pp. $57-81$.

[25] F. Rapetti, F. Dubois, and A. Bossavit, Integer matrix factorization for mesh defects detection, C. R. Acad. Sci. Paris Sér. I Math., 334 (2002), pp. 717-720.

[26] S. M. RaO, D. R. Wilton, and A. W. Glisson, Electromagnetic scattering by surfaces of arbitrary shape, IEEE Trans. Antennas and Propagation, 30 (1982), pp. 409-418.

[27] Z. REN AND A. RAZEK, Boundary edge elements and spanning tree technique in threedimensional electromagnetic field computation, Internat. J. Numer. Methods Engrg., 36 (1993), pp. 2877-2893.

[28] F.-X. Roux, lecture, University of Paris, Paris, France, 1984.

[29] J. Stillwell, Classical Topology and Combinatorial Group Theory, Grad. Texts in Math. 72, Springer-Verlag, New York, 1993

[30] F. L. TeiXeIRA AND W. C. Chew, Lattice electromagnetic theory from a topological viewpoint, J. Math. Phys., 40 (1999), pp. 169-187.

[31] H. Whitney, Geometric Integration Theory, Princeton University Press, Princeton, NJ, 1957. 